\title{
Resource Allocation in Millimeter-Wave Multicarrier-Division Duplex Systems with Hybrid Beamforming
}

\author{
Bohan Li, Lie-Liang Yang, Fellow, IEEE, Robert G. Maunder, Senior Member, IEEE, \\ and Songlin Sun, Senior Member, IEEE
}

\begin{abstract}
In-band full duplex (IBFD) systems require promising resource allocation (RA) strategies to fully exploit the available time-frequency resources. Furthermore, the acquisition of channel state information and signal reception in IBFD systems are significantly impacted by insufficient self-interference cancellation (SIC), impeding the applications of IBFD in practical wireless systems. Multicarrier-division duplex (MDD), which benefits low-budget SI mitigation in digital domain and flexible subcarrier assignment, is expected to be a promising transitional technique from half-duplex (HD) to IBFD. Hence, to demonstrate the advantages of MDD over HD, this paper first compares the upper bound performance of MDD and $\mathrm{HD}$ by applying unfair greedy RA. Then, considering a more complicated application scenario of the millimeter-wave (mmWave) with hybrid beamforming, we propose the RA optimization with the quality of service (QoS) constraints on both downlink (DL) and uplink (UL) mobile stations (MSs). To solve this non-convex RA problem, we divide it into a suboptimal subcarrier allocation problem, which is solved by the proposed improved fair greedy (IFG) algorithm, and a convex power allocation problem. Furthermore, we design two general hybrid precoder based on matrix factorization and direct approach, and a combiner having high SIC capability. Our results show that the proposed $\mathrm{RA}$ algorithm is capable of achieving the performance near the upper bound achieved by the unfair greedy algorithm, while concurrently guaranteeing the proportional fairness among all DL/UL MSs. The performance of the two precoding schemes is depended on the number of radio frequency chains supported. Finally, with appropriate antenna deployment, the proposed SIC algorithm is able to provide sufficient SI mitigation, and furthermore, can be implemented without impacting the RA operation.
\end{abstract}

Index Terms-Multicarrier-division duplex, MIMO, mmWave, resource allocation, hybrid beamforming, full duplex, selfinterference cancellation.

\section{INTRODUCTION}

With the increasing demand of high capacity and spectral efficiency (SE) for the next-generation wireless networks, two significant techniques have come into our vision, namely millimeter-wave (mmWave) and in-band full-duplex (IBFD) $[1,2]$. On one side, mmWave communication makes use of the unexploited spectrum resource with $\mathrm{GHz}$ of bandwidth

B. Li, L.-L. Yang and R. Maunder are with the School of Electronics and Computer Science, University of Southampton, SO17 1BJ, UK. (E-mail b12n18, lly, rm@ecs.soton.ac.uk, http://www-mobile.ecs.soton.ac.uk/lly). S Sun is with the School of Information and Communication Engineering, Beijing University of Posts and Telecommunications (BUPT). The project was supported in part by the EPSRC, UK, under Project EP/P034284/1 and in part by the Innovate UK project. to meet the requirements of high-rate applications. On the other side, owing to the downlink (DL) and uplink (UL) transmissions concurrently occurring on the same frequency band, IBFD has the potential to double the spectral efficiency that is achievable by the conventional half-duplex (HD) modes, such as frequency-division duplex (FDD) and time-division duplex (TDD) [2]. Moreover, the above two techniques can be joined to augment each other, so as to meet the challenges of the next generations of wireless communication systems [3].

However, it has been demonstrated in [4] that without an efficient method of self-interference (SI) suppression, IBFD can hardly outperform the conventional HD modes. To this end, various SI cancellation (SIC) techniques have been proposed, but most of which are however unable to provide enough SI reduction with the practical complexity for operation in largescale multiple-input multiple-output (MIMO) systems $[5,6]$. Therefore, to inherit the merits of IBFD while simultaneously circumvent the problem of SI, we proposed and investigated a multicarrier-division duplex multiuser MIMO (MDD/MUMIMO) system in [7]. According to [8], MDD is a transitional technique from HD to IBFD, enabling DL and UL to work within the same time and same frequency band but on different subcarriers. Owing to its feature, MDD has its natural advantage of SI suppression in digital domain aided by the fast Fourier transform (FFT) at receiver, which can cancel up to $50 \mathrm{~dB}$ of SI at nearly no cost of system resources [9]. Furthermore, compared with HD systems, since DL and UL signals in MDD systems are transmitted on the different subcarriers of an OFDM block, there are more degrees-offreedom (DOF) for dynamically allocating subcarriers to DL and UL, which further enhances spectral efficiency. Nonetheless, MDD belongs to the FD family. Hence, SI still needs to be sufficiently suppressed in the analog- and propagationdomain, so that the analog-to-digital conversion (ADC) at receiver can operate efficiently. Hence, in order to fully take the advantages of MDD, this paper will focus on the joint design of resource allocation (RA) and hybrid beamformers, with the consideration of quality of service $(\mathrm{QoS})$ constraints as well as practical SI suppression in MDD/MU-MIMO mmWave systems.

\section{A. Related Works}

With the increasing interest in MIMO systems, the joint design of beamformers and RA has become more and more 
attractive. In [10-12], the authors studied the channel and power allocation with digital beamforming and QoS constraints. However, the proposed approaches cannot be directly applied in the large-scale MIMO mmWave systems with hybrid beamforming. Furthermore, the algorithms with fairness constraint are unable to guarantee the proportional fairness exactly, leading to the performance fluctuation among users. The authors of [13] studied the RA to maximize the sum-rate of the mmWave OFDMA systems with hybrid beamforming under the power constraint of BS, where power allocation, precoder design and subcarrier assignment are concurrently implemented, causing a high complexity. Different from [13], the authors of [14] jointly optimized the hybrid beamformers and RA in a distributed way to minimize the transmission latency in mobile edge computing mmWave networks. However, the QoS requirements of users were not considered in both these references. Furthermore, in [15], a proportional fairness constrained hybrid beamforming algorithm was proposed for the DL transmission in mmWave MU-MIMO systems, in which the constant modulus constraints on analog beamformers are ignored for simplicity. Furthermore, the proposed algorithm fails to achieve the expected fairness, when the inter-user distances are large. We should note that in the resource allocation of mmWave systems, the QoS requirements of users are important, as users suffer from large path-losses, in particular, when they are at the cell edges. To the best of our knowledge, there is no work in the open literature considered the RA in the hybrid beamforming-assisted systems with the constraints on individual users' QoS.

On the other hand, there are limited works having so far studied the RA in FD OFDMA systems. In [16], the authors considered to maximize the sum-rate of a multiuser single-input single-output (MU-SISO) IBFD system by jointly optimizing the subcarrier assignment and power allocation. In [17], the joint user pairing and RA problem for MU-SISO IBFD system were investigated by proposing a low-complexity near-optimal matching algorithm. Moreover, the authors of [18] developed an optimal iterative RA algorithm, aiming at minimizing the total power consumption via jointly optimizing DL beamformer, UL transmit power and antenna selection. In [19], the authors jointly optimized the UL transmit power and DL beamforming to minimize the long-term transmit power under delay constraints. Although all the above-mentioned works endeavor to exploit the resources provided by FD, the SI problem, which is regarded as the biggest obstacle to achieve high-efficiency FD communications, has not been assumed in a practical way. More specifically, the SI was directly assumed as Gaussian noise in [16], while in [17], SI was treated as a coefficient in the range of $70 \mathrm{~dB}$ to $110 \mathrm{~dB}$. In [18] and [19], SI was assumed to be perfectly mitigated by a separate SIC subsystem before the beamforming design. To the authors' best knowledge, all the existing studies on the RA optimization in FD systems ignore the practical implementation of SIC, but assume a near-optimal SIC. This assumption may be feasible in a small-scale MIMO system equipped with a small number of antennas (or RF chains) supported by the conventional SIC approaches proposed, such as, in [20-22]. However, these SIC methods may cause significant power consumption and complexity in large-scale mmWave MIMO systems. Hence, a feasible RA algorithm for FD systems should be designed by considering a practically efficient SIC method, which is capable of providing sufficient SI suppression at the lowest possible cost of system resources.

\section{B. Contributions}

To fill the research gap, in this paper, we propose and study the RA in MDD/MU-MIMO mmWave systems for not only leveraging the DL/UL resources but also relieving the impact of SI, via designing hybrid beamforming and SIC schemes, while simultaneously meeting the fairness constraints nearperfectly. Our contributions can be summarized as follows:

- Firstly, considering a MU-SISO system with unfair greedy RA, we demonstrate the performance advantages of MDD against the HD modes of FDD and TDD.

- Secondly, we study the RA in MDD/MU-MIMO mmWave systems. Since MDD enables system to jointly allocate resources among DL/UL, which leads to a NPhard problem, we divide the joint UL/DL RA problem into the suboptimal subcarrier allocation and power allocation. Specifically for subcarrier allocation, an improved fair greedy (IFG) algorithm is proposed, which consists of three stages to concurrently achieve sum-rate maximization, coarse fairness and spatial multiplexing gain. After subcarrier allocation and applying the full-digital zero-forcing (ZF) precoding, power allocation becomes a convex solvable problem. Our results show that the proposed RA algorithms allow to attain the performance near the upper bound that is only achievable by the conventional unfair greedy algorithm. Simultaneously, our algorithms guarantee the near-accurate proportional fairness among all the DL/UL MSs, even when some MSs are located far away from BS.

- Thirdly, we design two hybrid precoding schemes for the implementation of RA based on the matrix factorization and direct approach, respectively, under the fairness constraint. Our studies show that although the matrix factorization method performs well in the systems without considering RA [23], it cannot perform as well as the direct approach, when the number of RF chains is relatively low. It is shown that this is because the difference between a full digital precoder and the hybrid precoder designed from it by using matrix factorization is magnified after RA, which leads to the increase of interuser interference.

- Furthermore, we propose an adaptive SIC algorithm in conjunction with the design of hybrid combiner at BS receiver. To the best of our knowledge, this is the first attempt to jointly consider SIC and RA in FD systems. It is shown that our SIC algorithm is capable of providing the flexible levels of SI suppression over a big range and decoupled with the implementation of RA.

The rest of the paper is organized as follows. In Section II, we address the modeling of the MDD/MU-MIMO mmWave system with hybrid beamforming and formulate the RA problem. Section III briefly compares MDD with the HD modes 
of FDD and TDD in the context of a MU-SISO system with unfair greedy RA. In Section IV, suboptimal subcarrier and power allocation are presented to optimize the sum rate and the fairness among DL and UL MSs. Two precoding strategies are proposed for RA in Section V, where an adaptive SIC method based on the design of BS combiner is also presented. Simulation results are presented and discussed in VI, and finally, conclusions are given in Section VII.

Throughout the paper, the following notations are used. $A, a, a$ : matrix, vector, scalar; $\mathcal{A},|\mathcal{A}|$ : set, cardinality of a set; $(\boldsymbol{a})_{i}: i$-th element of $\boldsymbol{a} ; \boldsymbol{A}^{(i,:)}, \boldsymbol{A}^{(:, j)}: i$-th row and $j$-th column of $A ;(A)_{i, j}:(i, j)$-th element of $A ;|A|, A^{*}, A^{T}$, $A^{-1}, A^{\dagger}, A^{H}$ : determinant, complex conjugate, transpose, inverse, pseudo-inverse, Hermitian transpose of $\boldsymbol{A} ; \arg (\boldsymbol{A})$ : phases of all elements of $A$. $\|\cdot\|_{2},\|\cdot\|_{F}$ : Euclidean norm of a vector, Frobenius norm of a matrix; $\boldsymbol{I}_{N}:(N \times N)$ identity matrix; $\mathcal{C N}(\mathbf{0}, \boldsymbol{A})$ : zero-mean complex Gaussian distribution with covariance matrix $A ; \operatorname{Tr}(\cdot), \log (\cdot), \mathbb{E}[\cdot]$ : trace, logarithmic and exception operators.

\section{SySTEM MODEL}

\section{A. MDD-Based Multiuser MIMO System Model}

Consider a MDD/MU-MIMO mmWave system, where an

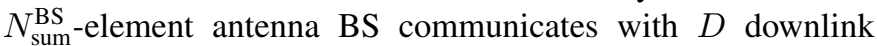
single-antenna MSs and $\bar{D}$ uplink single-antenna MSs, where $D+\bar{D}=D_{\text {sum. }}$. As shown in Fig. 1, we assume that BS implements the separate antenna configuration working in MDD mode, in which the Tx and Rx are equipped with $N$ and $\bar{N}$ antenna elements, respectively. By contrast, MSs work in HD mode. We further assume that the transmission channels between BS and MSs are frequency selective. In order to deal with small-scale fading, OFDM modulation is applied with the cyclic prefix introduced to avoid inter-symbol interference. In our proposed MDD system, all MSs choose the subcarriers from a set $\mathcal{M}$, with the size of $|\mathcal{M}|=M$. Denoting $\alpha_{m, d}$ and $\alpha_{m, \bar{d}}$ the two indicators of subcarrier assignments, which take values as

$\alpha_{m, d}\left(\right.$ or $\left.\alpha_{m, \bar{d}}\right)=\left\{\begin{array}{l}1, \text { if } d(\text { or } \bar{d}) \text { assigned subcarrier } m, \\ 0, \text { otherwise }\end{array}\right.$

where $d$ and $\bar{d}$ are DL MS and UL MS, respectively. Note that a subcarrier can only be assigned either to DL MSs or to UL MSs, not to both simultaneously. After subcarrier allocation, we obtain two mutually exclusive subsets, namely downlink subset $\mathcal{M}_{\mathrm{DL}}$ and uplink subset $\mathcal{M}_{\mathrm{UL}}$. The size of the two sets are pre-defined with considering the QoS requirements for both DL and UL MSs, and we have $\left|\mathcal{M}_{\mathrm{DL}}\right| \leq M_{\mathrm{DL}},\left|\mathcal{M}_{\mathrm{UL}}\right| \leq$ $M_{\mathrm{UL}}$ and $M_{\mathrm{DL}}+M_{\mathrm{UL}} \leq M$. Let the DL signal transmitted to $d$ or the UL signal transmitted by $\bar{d}$ on the $m$-th subcarrier be denoted as $z_{d}[m] \in \mathbb{C}$ or $z_{\bar{d}}[m] \in \mathbb{C}$. They satisfy $\mathbb{E}\left[\left|z_{d}[m]\right|^{2}\right]=\mathbb{E}\left[\left|z_{\bar{d}}[m]\right|^{2}\right]=1$ and $\mathbb{E}\left[z_{i}[m] z_{j}^{*}[m]\right]=0$, $\forall i \neq j$.

Although DL and UL are operated on different subcarriers, MDD-based system still suffers from SI in analog domain at BS, as IBFD systems [4]. If SI is not sufficiently suppressed in analog domain, the BS transmit signal may overwhelm

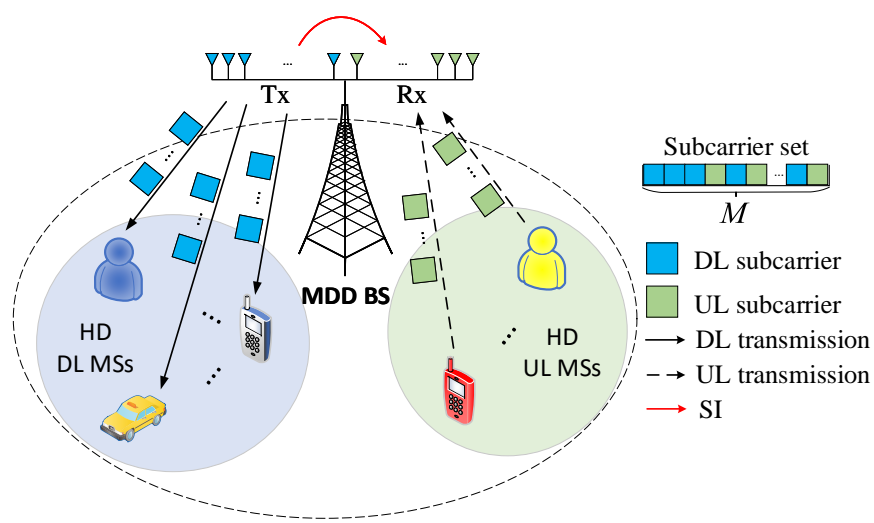

Fig. 1. System model: MDD/MU-MIMO mmWave network.

the ADC of the BS receiver and cause large unexpected quantization noise. Therefore, the effect of SI and its suppression will be considered in this paper. However, the inter-user interference from the UL MSs on the DL MSs is ignored. This is because in general, MSs are distributed from each other with relatively big distances, which results in big SI reduction due to propagation pathloss [2] $]^{1}$. Then, when all MSs and BS are assumed to be synchronized within an allowable limit, the interference among different subcarriers can be ideally canceled with the aid of the FFT operation in digital domain, as assumed in the references on IBFD OFDMA systems [16, 17].

\section{B. Channel Model}

1) Communication Channel: According to [25], we present a $T$-delay channel model combined with limited scattering characteristics of mmWave. As the antenna arrays at BS are separated, below we only introduce the wideband channel model between the BS transmit array and the $d$-th DL MS. The UL transmission channels have the same expression. The $t$-th delay tap of the DL channel, $\tilde{h}_{d}^{t} \in \mathbb{C}^{N}$, can be written as [25]

$$
\tilde{\boldsymbol{h}}_{d}^{t}=\beta \sum_{l=1}^{L_{d}} v_{l, d} p_{\mathrm{rc}, d}\left(t T_{s}-\tau_{l, d}\right) \alpha_{\mathrm{MS}, d}\left(\theta_{l, d}\right) \boldsymbol{\alpha}_{\mathrm{BS}}\left(\phi_{l, d}\right)
$$

where $L_{d}$ is the number of propagation paths, $\beta=$ $\sqrt{N /\left(L_{d} P_{L}\right)}, P_{L}, v_{l, d}, \tau_{l, d}, \theta_{l, d}, \phi_{l, d}$ are the pathloss, complex gain, delay, angle of arrival (AoA) and angel of departure (AoD) of the $l$-th path, respectively, $1 / T_{s}$ is the sampling frequency, $p_{\mathrm{rc}, d}(\tau)$ is the raised cosine (RC) pulse evaluated at the time of $\tau$. Finally, $\alpha_{\mathrm{MS}, d}\left(\theta_{l, d}\right)$ and $\boldsymbol{\alpha}_{\mathrm{BS}}\left(\phi_{l, d}\right)$ are the array response vectors of the receiver and the transmitter, respectively. Correspondingly, the $m$-th subcarrier channel can be expressed as

$$
\boldsymbol{h}_{d}[m]=\sum_{t=1}^{T} \tilde{\boldsymbol{h}}_{d}^{t} e^{-j 2 \pi t \frac{m}{M}}, m=1,2, \ldots, M
$$

\footnotetext{
${ }^{1}$ Note that, in the case where a UL MS is close to a DL MS, we can leverage the user scheduling operated at medium access control (MAC) layer to minimize the interference between these two MSs [24]. This is however out of the scope of this paper.
} 
2) Self-Interference Channel: We assume that the SI channel has both LOS component and NLOS component, which follows the expression of [26]

$$
\boldsymbol{H}_{\mathrm{SI}}=\sqrt{\frac{\kappa}{\kappa+1}} \boldsymbol{H}_{\mathrm{LOS}}+\sqrt{\frac{1}{\kappa+1}} \boldsymbol{H}_{\mathrm{NLOS}}
$$

where $\kappa$ is the Rician factor. The $(i, j)$-th element of $\boldsymbol{H}_{\mathrm{LOS}}$ satisfies an expression

$$
\boldsymbol{H}_{\mathrm{LOS}}(i, j)=\frac{\rho}{r_{i j}} \exp \left(-j 2 \pi \frac{r_{i j}}{\lambda}\right)
$$

where $\rho$ is the power normalization constant to make $\mathbb{E}\left[\left\|\boldsymbol{H}_{\mathrm{LOS}}\right\|_{F}^{2}\right]=N \bar{N}, r_{i j}$ denotes the distance between the $i$-th element of the BS transmit array and the $j$-th element of the $\mathrm{BS}$ receive array, $\lambda$ is the wavelength. Moreover, since the distance between the BS transmit array and the BS receive array is small, the NLOS components can be well modeled to experience flat fading, having an expression in the form of

$$
\boldsymbol{H}_{\mathrm{NLOS}}=\beta_{\mathrm{SI}} \sum_{l=1}^{L_{n}} v_{l} \boldsymbol{\alpha}_{\mathrm{R}}\left(\theta_{l}\right) \boldsymbol{\alpha}_{\mathrm{T}}^{\mathrm{H}}\left(\phi_{l}\right)
$$

where $L_{n}$ is the number of propagation paths between the $\mathrm{BS}$ transmit array and the $\mathrm{BS}$ receive array, while $\beta_{\mathrm{SI}}=$ $\sqrt{N \bar{N} / L_{n}}$. Additionally, $v_{l}, \boldsymbol{\alpha}_{\mathrm{R}}\left(\theta_{l}\right)$ and $\boldsymbol{\alpha}_{\mathrm{T}}^{\mathrm{H}}\left(\phi_{l}\right)$ have the similar definitions as that in (2).

\section{Downlink Transmission}

For DL transmission, the signal $z_{d}[m]$ is first processed by a digital precoder $\boldsymbol{f}_{\mathrm{BB}}^{d}[m] \in \mathbb{C}^{N_{\mathrm{RF}}}$, where $N_{\mathrm{RF}}$ denotes the number of RF chains at BS transmitter. Then, after DAC operation and RF processing, the time-domain signal is further processed by an analog precoder $\boldsymbol{F}_{\mathrm{RF}} \in \mathbb{C}^{N \times N_{\mathrm{RF}}}$. As analog beamformers are implemented using unit-modulus phase shifters, each element in $\boldsymbol{F}_{\mathrm{RF}}$ should meet the constraint $\left|\left(\boldsymbol{F}_{\mathrm{RF}}\right)_{i, j}\right|=1$. Finally, the signal $\boldsymbol{x}_{d}[m]$ transmitted on the $m$ th subcarrier by the BS transmit antennas can be formulated as

$$
\boldsymbol{x}_{d}^{\mathrm{DL}}[m]=\alpha_{m, d} \sqrt{p_{m, d}} \boldsymbol{F}_{\mathrm{RF}} \boldsymbol{f}_{\mathrm{BB}}^{d}[m] z_{d}[m], m=1, \ldots, M
$$

where $p_{m, d}$ denotes the transmit power for MS $d$ over subcarrier $m$, satisfying $\sum_{d=1}^{D} \sum_{m=1}^{M} p_{m, d} \leq P_{\mathrm{DL}}$ wiht $P_{\mathrm{DL}}$ denoting the total power of DL transmission. Moreover, we normalize $\left\|\boldsymbol{F}_{\mathrm{RF}} \boldsymbol{f}_{d}[m]\right\|_{2}^{2}=1$ to ensure that $\left\|\boldsymbol{x}_{d}^{\mathrm{DL}}[m]\right\|_{2}^{2}=\alpha_{m, d} p_{m, d}$. Given the $m$-th subchannel vector $\boldsymbol{h}_{d}[m] \in \mathbb{C}^{N}$ to the $d$-th $\mathrm{MS}$, the received signal at MS $d$ is given by

$$
y_{d}^{\mathrm{DL}}[m]=\boldsymbol{h}_{d}^{H}[m] \sum_{d=1}^{D} \boldsymbol{x}_{d}^{\mathrm{DL}}[m]+n_{d}[m]
$$

where $n_{d}[m] \sim \mathcal{C N}\left(0, \sigma^{2}\right)$ is the complex Gaussian noise added to the $m$-th subcarrier, which has the power of $\sigma^{2}$.

It is assumed that coherent detection is employed at MSs. The signal-to-interference-plus-noise ratio (SINR) of the $m$-th subcarrier at the $d$-th DL MS can be obtained from (8) and is given by

$$
\operatorname{SINR}_{m, d}=\frac{\alpha_{m, d} p_{m, d}\left|\boldsymbol{h}_{d}^{H}[m] \boldsymbol{F}_{\mathrm{RF}} \boldsymbol{f}_{\mathrm{BB}}^{d}[m]\right|^{2}}{\sum_{i \neq d}^{D} \alpha_{m, i} p_{m, i}\left|\boldsymbol{h}_{d}^{H}[m] \boldsymbol{F}_{\mathrm{RF}} \boldsymbol{f}_{\mathrm{BB}}^{i}[m]\right|^{2}+\sigma^{2}}
$$

Furthermore, when Gaussian distributed transmit signals are assumed, the achievable sum-rate of the $d$-th DL MS is given by $R_{d}=\sum_{m=1}^{M} \log \left(1+\operatorname{SINR}_{m, d}\right)$.

\section{Uplink Transmission}

For UL transmission, the multiple-access signal received from the $m$-th subcarrier by BS receiver can be expressed as

$$
\boldsymbol{y}_{\mathrm{UL}}[m]=\underbrace{\sum_{\bar{d}=1}^{\bar{D}} \boldsymbol{x}_{\bar{d}}^{\mathrm{UL}}[m]}_{\text {Desired signal }}+\underbrace{\boldsymbol{H}_{\mathrm{SI}} \boldsymbol{x}_{\mathrm{DL}}}_{\mathrm{SI}}+\boldsymbol{n}[m]
$$

where $x_{\mathrm{DL}}=\sum_{d=1}^{D} \sum_{m=1}^{M} \boldsymbol{x}_{d}^{\mathrm{DL}}[m]$ generates SI, the desired UL signal from MS $\bar{d}$ is $\boldsymbol{x}_{\bar{d}}^{\mathrm{UL}}[m]=\sqrt{p_{m, \bar{d}}} \alpha_{m, \bar{d}} \boldsymbol{h}_{\bar{d}}[m] z_{\bar{d}}[m]$, where $p_{m, \bar{d}}$ denotes the transmit power on subcarrier $m$ of the $\bar{d}$-th UL MS. The transmit power of a UL MS satisfies the constraint of $\sum_{m=1}^{M} \alpha_{m, \bar{d}} p_{m, \bar{d}} \leq P_{\bar{d}}$. Finally, $\boldsymbol{n}[m] \sim \mathcal{C N}\left(\mathbf{0}, \sigma^{2} \boldsymbol{I}_{\bar{N}}\right)$ is the Gaussian noise.

At the BS receiver, the received signal of (10) is first

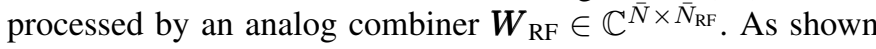
in (10), the received signal includes both SI and desired UL signal. In theory, the SI can be removed in digital domain by FFT operation, as UL and DL signals are transmitted on different subcarriers. However, the power of SI may be much larger than the power of desired signal. Hence, SI may overwhelm the limited dynamic range of the ADC at BS receiver, introducing significant quantization noise. Therefore, after the transmitter preprocessing seen in (7) and the receiver combining using $W_{\mathrm{RF}}$, SI should be sufficiently suppressed to make the received desired signal pass the ADC with high efficiency. As done in [20], we model the residual $\mathrm{SI}^{2}$, by a vector $\boldsymbol{r}_{\mathrm{SI}} \sim \mathcal{C N}\left(\mathbf{0}, \xi \operatorname{diag}\left(\mathbb{E}\left[\boldsymbol{W}_{\mathrm{RF}}^{H} \boldsymbol{H}_{\mathrm{SI}} \boldsymbol{x}_{\mathrm{DL}} \boldsymbol{x}_{\mathrm{DL}}^{H} \boldsymbol{H}_{\mathrm{SI}}^{H} \boldsymbol{W}_{\mathrm{RF}}\right]\right)\right)$, where $0<\xi \leq 1$ denotes the SIC capability provided by the SIC techniques implemented in propagation- and analogdomain, such as those considered in $[2,6,21]$. Consequently, after ADC conversion, FFT operation and digital combining, the final signal for detecting the information of MS $\bar{d}$ can be expressed as

$$
\begin{aligned}
y_{\bar{d}}[m]= & \boldsymbol{w}_{\mathrm{BB}}^{\bar{d}, H}[m] \boldsymbol{W}_{\mathrm{RF}}^{H} \sum_{\bar{d}=1}^{\bar{D}} \boldsymbol{x}_{\bar{d}}^{\mathrm{UL}}[m] \\
& +\boldsymbol{w}_{\mathrm{BB}}^{\bar{d}, H}[m] \boldsymbol{W}_{\mathrm{RF}}^{H} \boldsymbol{n}[m]+\boldsymbol{w}_{\mathrm{BB}}^{\bar{d}, H}[m] \boldsymbol{r}_{\mathrm{SI}}
\end{aligned}
$$

where $\boldsymbol{w}_{\mathrm{BB}}^{\bar{d}}[m] \in \mathbb{C}^{\bar{N}_{\mathrm{RF}}}$ is the digital combiner for detecting the $m$ th subcarrier of the $\bar{d}$-th UL MS. Note furthermore

\footnotetext{
${ }^{2}$ Note that the residual SI consists of the combined effect of the additive noise introduced by automatic gain control (AGC), non-linearity of ADC and the phase noise generated by oscillator due to RF imperfection [20].
} 
that $\boldsymbol{W}_{\mathrm{RF}}$ satisfies $\left|\left(\boldsymbol{W}_{\mathrm{RF}}\right)_{i, j}\right|=1$, as it operates the analog beamforming.

Similar to the DL detection, the SINR achieved by the $m$ th subcarrier of the $\bar{d}$-th UL MS is given by

$$
\begin{aligned}
\operatorname{SINR}_{m, \bar{d}} & =\frac{\alpha_{m, \bar{d}} p_{m, \bar{d}}\left|\boldsymbol{w}_{\mathrm{BB}}^{\bar{d}, H}[m] \boldsymbol{W}_{\mathrm{RF}}^{H} \boldsymbol{h}_{\bar{d}}[m]\right|^{2}}{\sum_{j \neq \bar{d}}^{\bar{D}} \alpha_{m, j} p_{m, j}\left|\boldsymbol{w}_{\mathrm{BB}}^{\bar{d}, H}[m] \boldsymbol{W}_{\mathrm{RF}}^{H} \boldsymbol{h}_{j}[m]\right|^{2}+I N_{m, \bar{d}}} \\
I N_{m, \bar{d}} & =\sigma^{2}\left\|\boldsymbol{w}_{\mathrm{BB}}^{\bar{d}, H}[m] \boldsymbol{W}_{\mathrm{RF}}^{H}\right\|_{2}^{2}+\left|\boldsymbol{w}_{\mathrm{BB}}^{\bar{d}, H}[m] \boldsymbol{r}_{\mathrm{SI}}\right|_{2}^{2}
\end{aligned}
$$

Correspondingly, the data-rate achieved by the $\bar{d}$-th UL MS is given by $\bar{R}_{\bar{d}}=\sum_{m=1}^{M} \log \left(1+\operatorname{SINR}_{m, \bar{d}}\right)$.

\section{E. Problem Formulation}

To design an optimum adaptive RA scheme achieving proportional data rate constraints in MDD/MU-MIMO system with hybrid beamforming, we have to solve a non-convex problem described as

$$
\begin{array}{ll} 
& \max _{\text {s.t. }} \quad \sum_{d=1}^{D} \sum_{m=1}^{M} \alpha_{m, d} p_{m, d} \leq P_{\mathrm{DL}} \\
& \sum_{m=1}^{M} \alpha_{m, \bar{d}} p_{m, \bar{d}} \leq P_{\bar{d}}, \quad \forall \bar{d} \\
& \alpha_{m, d}+\alpha_{m, \bar{d}} \leq 1, \quad \forall m, d, \bar{d} \\
& \sum_{d=1}^{D} \alpha_{m, d} \leq N_{\mathrm{RF}}, \quad \forall m \\
& \sum_{\mathrm{D}} \alpha_{m, \bar{d}} \leq \bar{N}_{\mathrm{RF}}, \quad \forall m \\
& { }_{\bar{d}=1} \alpha_{\mathrm{RF}}[m], \boldsymbol{w}_{\mathrm{BB}}[m], p_{m, d}, p_{m, \bar{d}} \\
& R_{1}: R_{2}: \ldots: R_{D}=\gamma_{1}: \gamma_{2}: \ldots: \gamma_{D} \\
& \bar{R}_{1}: \bar{R}_{2}: \ldots: \bar{R}_{\bar{D}}=\eta_{1}: \eta_{2}: \ldots: \eta_{\bar{D}} \\
& \left\|\boldsymbol{F}_{\mathrm{RF}} \boldsymbol{f}_{\mathrm{BB}}^{d}[m]\right\|_{2}^{2}=1, \forall m, d \\
& \left|\left(\boldsymbol{F}_{\mathrm{RF}}\right)_{i, j}\right|=\left|\left(\boldsymbol{W}_{\mathrm{RF}}\right)_{i, j}\right|=1, \quad \forall i, j \\
& \left|\mathcal{M}_{\mathrm{DL}}\right| \leq M_{\mathrm{DL}},\left|\mathcal{M}_{\mathrm{UL}}\right| \leq M_{\mathrm{UL}}
\end{array}
$$

where $\Lambda=\sum_{\forall m, d, \bar{d}} \log _{2}\left(1+\operatorname{SINR}_{m, d}\right)+\log _{2}\left(1+\operatorname{SINR}_{m, \bar{d}}\right)$, (13b) and (13c) impose the constraint on the transmit power at BS and individual UL MSs, respectively, (13d) explains that one subcarrier can only be assigned either to DL or to UL, (13e) and (13f) state that the number of MSs allocated to the same subcarrier does not exceed the number of RF chains of the DL and UL, respectively, $\left\{\gamma_{i}\right\}_{i=1}^{D}$ in (13g) and $\left\{\eta_{i}\right\}_{i=1}^{\bar{D}}$ in (13h) are given to ensure the proportional fairness among DL and UL $\mathrm{MSs}^{3}$. Finally, (13k) means that the number of DL and UL subcarriers have their specific constraints.

\footnotetext{
${ }^{3}$ Note that the exact proportional fairness shown in (13h) cannot be guaranteed among UL MSs in principle. This is because UL MSs work in the distributed manner and carry out power allocation separately. Therefore, the proportional fairness of UL can only be implemented via subcarrier allocation.
}

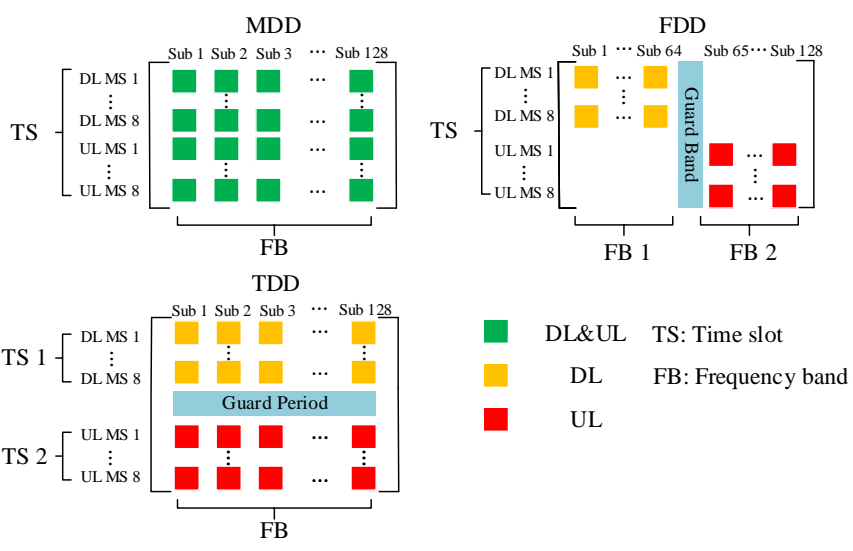

Fig. 2. Illustration of subcarrier resources in the MDD, TDD and FDD modes, where green squares denote the subcarriers available for both DL and UL allocations, yellow squares denote the subcarriers only for DL allocations, while red squares represent the subcarriers only for UL allocations.

\section{Potential of MDD with Resource Allocation}

Before attempting to solve the optimization problem (13), let us first demonstrate the potential of MDD over the HD modes of FDD and TDD, when the unfair greedy (UG) RA algorithm is employed ${ }^{4}$. To make the comparison straightforward, here we assume a MU-SISO system, where both BS and MS terminals employ single antenna, and the channel models of (2) with $P_{L}=1$. We assume that the transmit power of BS is $P_{\mathrm{DL}}=D$, and that of each UL MS is $P_{\bar{d}}=1$. The noise variance for both DL and UL is $\sigma^{2}=1 / \mathrm{SNR}$. Furthermore, as we compare the upper-bound of the three schemes, we assume that there is no SI in MDD system or in other words, ideal SIC is implemented.

Fig. 2 illustrates the subcarriers available for UL/DL allocation, when MDD, FDD and TDD are respectively employed. We can observe that as the UL/DL in MDD mode are operated within the same frequency band at the same time, a subcarrier can be assigned either to DL or to UL. By contrast, in FDD mode (or TDD mode), DL and UL MSs are constrained on different frequency bands (or time slots). Hence, a subcarrier is pre-defined either for DL or for UL, and cannot be allocated by jointly considering both the UL and DL MSs.

To compare these modes, we assume that both DL and UL are assigned an equal resource in TDD and FDD modes, in terms of the number of time slots and subcarriers, respectively. To match this, when MDD mode is considered, we assume that DL and UL have the same number of subcarriers. Hence, the spectral efficiency of the MDD-, FDD- and TDD-based

\footnotetext{
${ }^{4}$ To demonstrate the upper bound performance, the UG RA algorithm is employed, which maximizes the sum-rate of OFDM systems [27].
} 


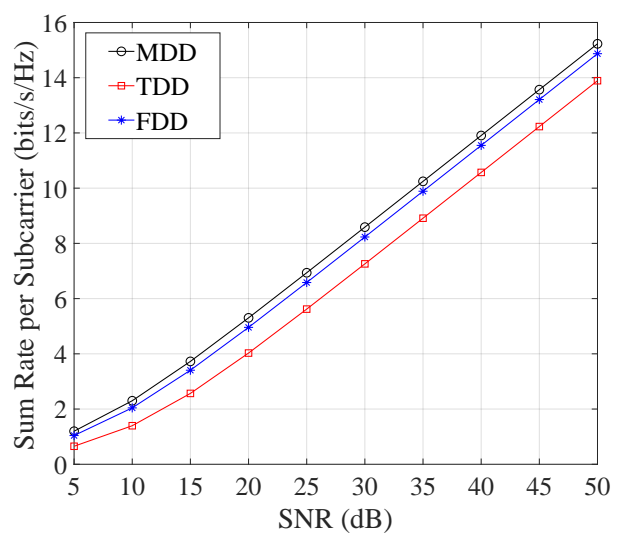

(a) Sum rate vs. SNR

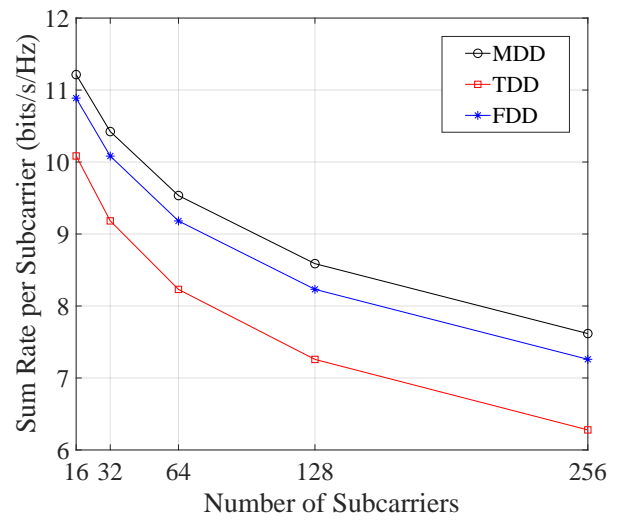

(b) Sum rate vs. number of subcarriers

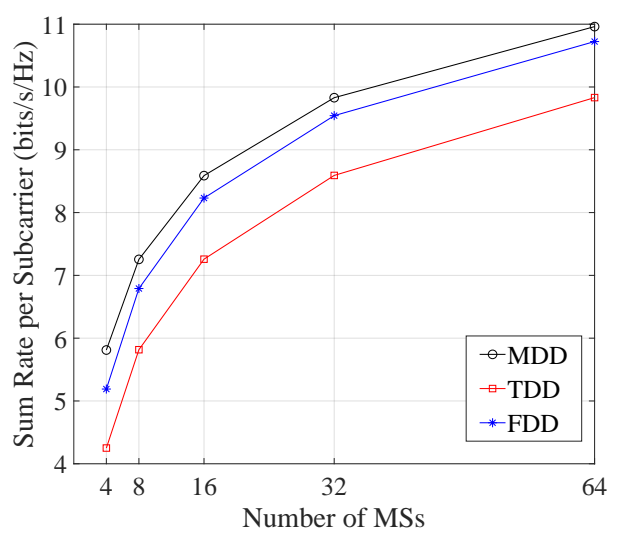

(c) Sum rate vs. number of MSs

Fig. 3. Sum rate achieved by the MDD-, FDD- and TDD-based MU-SISO systems. The experimental parameters in three subfigures are: (a) $M=128$, $D=8$ and $\bar{D}=8$; (b) $\mathrm{SNR}=30 \mathrm{~dB}, D=8$ and $\bar{D}=8$; (c) $\mathrm{SNR}=30$ $M=128$ and $D=\bar{D}$

MU-SISO systems can be expressed as

$$
\begin{aligned}
R_{\mathrm{MDD}} & =\frac{\alpha_{\mathrm{MDD}}}{M} \sum_{\tilde{m}=1}^{M} R_{\tilde{m}}, \\
R_{\mathrm{FDD}} & =\frac{\alpha_{\mathrm{FDD}}}{M}\left(\sum_{m=1}^{M / 2} R_{m}+\sum_{\bar{m}=M / 2}^{M} R_{\bar{m}}\right), \\
R_{\mathrm{TDD}} & =\frac{\alpha_{\mathrm{TDD}}}{M}\left(\sum_{m=1}^{M} R_{m}+\sum_{\bar{m}=1}^{M} R_{\bar{m}}\right)
\end{aligned}
$$

where $m$ and $\bar{m}$ denote the corresponding subcarriers assigned to DL and UL, respectively, $\tilde{m} \in\{m, \bar{m}\}$. Note that, as both DL and UL are always active in MDD and FDD modes while in TDD mode, DL and UL become active alternatively, we have $\alpha_{\mathrm{MDD}}=\alpha_{\mathrm{FDD}}=2 \alpha_{\mathrm{TDD}}=1$. In our simulations for Fig. 3 , we set $M=128, D=\bar{D}=8$.

From Fig. 3 we can observe that MDD is capable of outperforming both TDD and FDD. The reason behind is that, in MDD mode, each subcarrier has the largest spatialdomain degree-of-freedoms (DoFs) (i.e., 16) to be allocated either to a DL or a UL MS. By contrast, in TDD or FDD mode, as the $8 \mathrm{UL}$ MSs and the 8 DL MSs cannot jointly share the resources, each subcarrier has only 8 DoFs to be allocated. Additionally, we should mention that the SI in MDD and the effect of guard period/band in TDD/FDD, as shown in Fig. 2, are ignored in the comparison considered in Fig. 3, so as to focus on the upper-bound performance of the three modes. Furthermore, it is worth noting that in practice, the SI in such a MU-SISO MDD system can be perfectly suppressed by invoking an existing propagation/analog-domain SIC approach followed by the FFT operation in digital domain [22]. By contrast, the guard period/band used in TDD/FDD are indispensable. Therefore, we can be implied that the performance gap between MDD and TDD/FDD, as depicted in Fig. 3, may be widened in practice.

However, it is noteworthy that the performance advantage of MDD is attained under the assumption of the sharedantenna configuration, which may be degraded in the separateantenna configuration resulted from the loss of antenna gain, when MDD and TDD/FDD modes assume the same number of antenna elements. Nevertheless, the studies show that the separate-antenna configuration is more desirable in FD systems, since it has the potential to suppress SI at lower cost than the shared-antenna configuration [28]. On the other side, when the large antenna arrays satisfying $\left(N+\bar{N} \gg D_{\text {sum }}\right)$ are considered, we have the massive MIMO structure that is envisioned for future wireless systems. In this scenario, the loss of antenna gain and spatial multiplexing gain in the MDD systems resulted from separate-antenna configuration can be negligible [29].

In addition, we should also note that the implementation of RA at BS requires near accurate CSI and it is usually a knotty challenge in high-mobility scenarios. For instance, when a car moving at a speed of $36 \mathrm{~km} / \mathrm{h}$ communicates within $30 \mathrm{GHz}$ mmWave band, the normalized Doppler spread is 0.07 (when assuming $15 \mathrm{kHz}$ inter-subcarrier spacing). Consequently, channels vary fast from symbol to symbol, according to the Jakes autocorrelation model [30], which may lead to the outdated CSI for DL signal preprocessing after the UL training, when TDD systems are considered. To mitigate this problem, extra training pilots have to be added to the phase of data transmissions. However, for a given coherence period, increasing UL pilots not only directly results in the decrease of UL data rate, but also the degradation of DL data rate, as UL training has to be executed more frequently. As for FDD, it is well-known that it is infeasible for operation in massive MIMO systems, due to the extra complexity of CSI acquisition and the extreme overhead introduced by DL training and the 
CSI feedback from MSs to BS, not to mention the channel aging resulted from CSI estimation and feedback.

By contrast, in MDD-based systems, both DL and UL are operated within the same time slot and also the same frequency band. Hence, after the normal (initial) UL training, BS can concurrently update the CSI with the aid of either the pilots sent along with the UL data or the decision-directed channel estimation using the detected UL data [31]. Therefore, MDD systems are capable of implementing the DL transmission based on the CSI of nearly real-time. In other words, MDD can effectively avoid the channel aging problem. Note that above is just a brief comparison between MDD and TDD/FDD, as it is beyond the scope of this paper. A comprehensive comparison of MDD and TDD/FDD in fast fading environments will be provided in another paper in the near future.

\section{Subcarrier And Power Allocation}

In order to approximately solve the non-convex and NP-hard problem described in (13), we seek the suboptimal methods to divide the optimization into subcarrier allocation and power allocation. To be more specific, the variables $\left\{\alpha_{m, d}, \alpha_{m, \bar{d}}\right\}$ in (13a) are first obtained from the subcarrier allocation based on an improved fair greedy (IFG) algorithm, followed by the power allocation, for which the values of $p_{m, d}$ are calculated by solving a convex optimization problem while that of $p_{m, \vec{d}}$ are obtained by the water-filling algorithm. Note that in this section, we assume that the system is nearly free from SI (i.e., $\xi \ll 1$ ), as done in the other IBFD RA systems [17, 18]. However, different from these references, in this paper, we will propose an adaptive beamforming based SIC method in Section V, which guarantees that SI has little impact on the performance of the RA in this section ${ }^{5}$.

\section{A. Subcarrier Allocation}

Let $\mathcal{M}_{d}$ and $\mathcal{M}_{\bar{d}}$ denote the sets of subcarriers assigned to the $d$-th DL MS and $\bar{d}$-th UL MS, respectively. Hence, we have $\mathcal{M}_{\mathrm{DL}}=\mathcal{M}_{1} \cup \ldots \cup \mathcal{M}_{d} \ldots \cup \mathcal{M}_{D}$ and $\mathcal{M}_{\mathrm{UL}}=$ $\mathcal{M}_{1} \cup \ldots \cup \mathcal{M}_{\bar{d}} \ldots \cup \mathcal{M}_{\bar{D}}$. Let $\mathcal{D}_{m}$ (or $\overline{\mathcal{D}}_{m}$ ) denotes the set of DL MSs (or UL MSs), which are allocated the $m$-th subcarrier. $\mathcal{D}_{m}$ (or $\overline{\mathcal{D}}_{m}$ ) satisfies $\left|\mathcal{D}_{m}\right| \leq N_{\mathrm{RF}}$ (or $\left|\overline{\mathcal{D}}_{m}\right| \leq \bar{N}_{\mathrm{RF}}$ ). Note again that the same subcarrier $m$ can only be assigned either to DL or to UL, not to both simultaneously. During the subcarrier allocation stage, we assume that the maximal-ratio transmission (MRT) assisted precoding is employed by BS for its computational simplicity [29]. Then, the SNR for the DL and UL MSs can be expressed as $\operatorname{SNR}_{m, d}=\frac{\left\|\boldsymbol{h}_{d}[m]\right\|_{2}^{2}}{\sigma^{2}}$ and $\mathrm{SNR}_{m, \bar{d}}=\frac{\left\|\boldsymbol{h}_{\bar{d}}[m]\right\|_{2}^{2}}{\sigma^{2}}$, respectively. Correspondingly, the achievable rates of the DL MS $d$ and UL MS $\bar{d}$ can be temporarily expressed as $\tilde{R}_{d}=\sum_{m \in \mathcal{M}_{\mathrm{d}}} \log \left(1+\mathrm{SNR}_{m, d}\right)$ and $\tilde{R}_{\bar{d}}=\sum_{m \in \mathcal{M}_{\bar{d}}} \log \left(1+\mathrm{SNR}_{m, \bar{d}}\right)$.

${ }^{5}$ To the best of our knowledge, there are no SIC methods in hybrid beamforming systems, which can provide more than $100 \mathrm{~dB}$ of SIC at low complexity and low power consumption. Furthermore, we found that there are some beamforming based SIC approaches, such as that studied in [26, 32], which are coupled with RA. With these approaches, the results of RA change during the process of SI suppression.

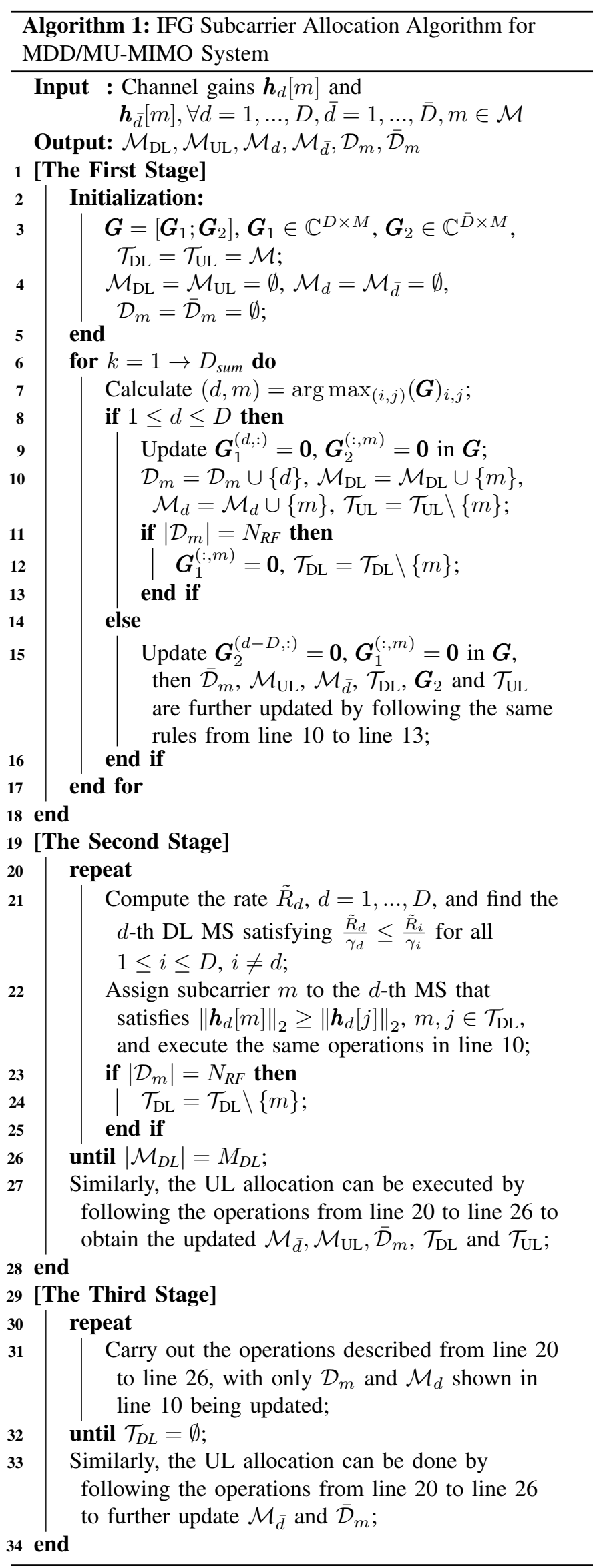


Then, the IFG subcarrier allocation algorithm is operated, which enables MSs to choose the best possible subchannels and exploit the space degree-of-freedom in MIMO systems while under the constraint of fairness. The proposed IFG subcarrier allocation algorithm is stated as Algorithm 1, which is divided into three stages. In view of the practice that DL transmission usually needs more resources than UL transmission, the algorithm commences allocating subcarriers to DL MSs during all of the three stages. Specifically, during the first stage one subcarrier is initially assigned to each MS by employing the UG subcarrier allocation algorithm. During the second stage, subcarriers are allocated with considering the fairness until the constraint (13k) is satisfied. Finally, the third stage leverages the spatial diversity provided by the MIMO system to accomplish the rest allocation. The details of the three stages of Algorithm 1 are stated as follows:

1) During the first stage, the channel gain matrix, $G=$ $\left[\boldsymbol{G}_{1} ; \boldsymbol{G}_{2}\right]$, is initialized to $g_{i j}=\left\|\boldsymbol{h}_{i}[j]\right\|_{2}$, where $\boldsymbol{G}_{1} \in$ $\mathbb{C}^{D \times M}, \boldsymbol{G}_{2} \in \mathbb{C}^{\bar{D} \times M}$. The candidate subcarrier set is initialized to $\mathcal{T}_{\mathrm{DL}}=\mathcal{M}$ for $\mathrm{DL}$ and to $\mathcal{T}_{\mathrm{UL}}=\mathcal{M}$ for UL. Furthermore, the iteration index is set to $k=1$. During the $k$-th iteration, the largest element $(\boldsymbol{G})_{d, m}$ of the remaining candidates of $\boldsymbol{G}$ is identified. Depending on MS $d$ being a DL MS or a UL MS, there are two options to update the related sets, as shown in line 9 and line 15 . Note that, since each MS is only assigned one subcarrier during the first stage, the $d$-th row is removed from $G$ after the $d$-th MS is assigned a subcarrier during the $k$-th iteration. Simultaneously, under the constraint of (13d) that a subcarrier assigned to DL (or UL) can no longer be assigned to UL (or DL), the $m$-th column is also removed from $\boldsymbol{G}_{2}$ (or $\boldsymbol{G}_{1}$ ), after the $m$-th subcarrier is assigned during the $k$ th iteration. Furthermore, to meet the constraints of (13e) and (13f), if a subcarrier has already been assigned to $N_{\mathrm{RF}}$ DL MSs (or $\bar{N}_{\mathrm{RF}} \mathrm{UL}$ $\mathrm{MSs}$ ), the subcarrier and its corresponding column are removed from both $\mathcal{T}_{\mathrm{DL}}$ (or $\mathcal{T}_{\mathrm{UL}}$ ) and $\boldsymbol{G}_{1}$ (or $\boldsymbol{G}_{2}$ ).

2) During the second stage, subcarriers are allocated with the consideration of fairness. For this purpose, during an allocation iteration, the $d$-th DL MS with the lowest ratio of $\tilde{R}_{d} / \gamma_{d}$ first chooses the best subcarrier $m$ from the candidate set $\mathcal{T}_{\mathrm{DL}}$, as described in line 21 . Then, $\tilde{R}_{d}$ is updated before going to the next iteration. This procedure is repeated until the condition $\left|\mathcal{M}_{\mathrm{DL}}\right|=M_{\mathrm{DL}}$ is satisfied. Analogously, UL allocation can be executed.

3) After the second stage of allocation, all MSs (including both DL and UL MSs) have obtained their subcarriers. However, the conditions of (13e) and (13f) with equality might not be reached. This implies that there are still spatial degrees-of-freedom that can be exploited. Therefore, during the third stage, subcarrier allocation following the second stage is continued, until the constraints of (13e) and (13f) with equality are reached.

It can be shown that after subcarrier allocation, the fairness constraints of (13g) and (13h) can be coarsely accomplished. Next, power allocation is executed to attain the proportional rate constraints of $(13 \mathrm{~g})$ and $(13 \mathrm{~h})$, while maximizing the total rate of the system, as detailed below.

\section{B. Power Allocation}

After subcarrier allocation, the optimization problem in (13) can be divided into the DL power allocation and UL power allocation, which can be executed independently.

Specifically, for DL, the power allocation problem can now be stated as

$$
\begin{aligned}
& \max _{\boldsymbol{F}_{\mathrm{RF}}, \boldsymbol{f}_{\mathrm{BB}}^{d}[m], p_{m, d}} \sum_{m \in \mathcal{M}_{\mathrm{DL}}} \sum_{d \in \mathcal{D}_{m}}\left(1+\frac{p_{m, d}\left|\boldsymbol{h}_{d}^{H}[m] \boldsymbol{F}_{\mathrm{RF}} \boldsymbol{f}_{\mathrm{BB}}^{d}[m]\right|^{2}}{\sum_{i \in \mathcal{D}_{m}, i \neq d} p_{m, i}\left|\boldsymbol{h}_{d}^{H}[m] \boldsymbol{F}_{\mathrm{RF}} \boldsymbol{f}_{\mathrm{BB}}^{i}[m]\right|^{2}+\sigma^{2}}\right) \\
& \log _{2}(13 g),(13 i),(13 j) \\
& \text { s.t. }(13) \\
& \sum_{d=1}^{D} \sum_{m \in \mathcal{M}_{d}} p_{m, d} \leq P_{\mathrm{DL}}
\end{aligned}
$$

By contrast, for UL, the power allocation problem can be formulated as

$$
\begin{aligned}
& \max _{p_{m, \bar{d}}} \sum_{m \in \mathcal{M}_{\mathrm{UL}}} \sum_{\bar{d} \in \overline{\mathcal{D}}_{m}} \\
& \log _{2}\left(1+\frac{p_{m, \bar{d}}\left|\boldsymbol{w}_{\mathrm{BB}}^{\bar{d}, H}[m] \boldsymbol{W}_{\mathrm{RF}}^{H} \boldsymbol{h}_{\bar{d}}[m]\right|^{2}}{\sum_{j \in \overline{\mathcal{D}}_{m}, j \neq \bar{d}} p_{m, j}\left|\boldsymbol{w}_{\mathrm{BB}}^{\bar{d}, H}[m] \boldsymbol{W}_{\mathrm{RF}}^{H} \boldsymbol{h}_{j}[m]\right|^{2}+I N_{m, \bar{d}}}\right) \\
& \text { s.t. } \quad \sum_{m \in \mathcal{M}_{\bar{d}}} p_{m, \bar{d}} \leq P_{\bar{d}}, \quad \forall \bar{d}
\end{aligned}
$$

Observing from (15) and (16), we can know that DL power allocation is under the constraint of the total power of all DL MSs. By contrast, each UL MS carries out power allocation separately under the constraint of individual MS's power. Below we first consider DL power allocation.

1) Downlink Power Allocation: The optimization problem in (15) is the sum-rate maximization problem coupled with fairness constraints, which is still a non-convex problem. To simplify it, we first apply the ZF precoding with assuming a fixed power assignment to all MSs so as to obtain a fulldigital precoder. This will allow us to transfer the non-convex problem of (15) to a convex optimization problem. To this objective, the full-digital precoder can be expressed as

$$
\boldsymbol{F}_{\mathrm{ZF}}[m]=\boldsymbol{H}_{\mathrm{DL}}^{H}[m]\left(\boldsymbol{H}_{\mathrm{DL}}[m] \boldsymbol{H}_{\mathrm{DL}}^{H}[m]\right)^{-1}
$$

where $\quad \boldsymbol{H}_{\mathrm{DL}}[m] \quad \in \quad \mathbb{C}^{\left|\mathcal{D}_{m}\right| \times N} \quad=$ $\left[\boldsymbol{h}_{1}[m], \ldots, \boldsymbol{h}_{d}[m], \ldots, \boldsymbol{h}_{\left|\mathcal{D}_{m}\right|}[m]\right]^{H}$, and $\boldsymbol{F}_{\mathrm{ZF}}[m] \in \mathbb{C}^{N \times\left|\mathcal{D}_{m}\right|}=$ $\left[\boldsymbol{f}_{\mathrm{ZF}}^{1}[m], \ldots, \boldsymbol{f}_{\mathrm{ZF}}^{d}[m], \ldots, \boldsymbol{f}_{\mathrm{ZF}}^{\left|\mathcal{D}_{m}\right|}[m]\right]$. It can be shown that owing to the power constraint in $(15 \mathrm{~d}), \boldsymbol{f}_{\mathrm{ZF}}^{d}[m]$ is in fact a normalized version, given as $\boldsymbol{f}_{\mathrm{ZF}}^{d}[m] \leftarrow \boldsymbol{f}_{\mathrm{ZF}}^{d}[m] /\left\|\boldsymbol{f}_{\mathrm{ZF}}^{d}[m]\right\|_{2}$. 
Consequently, upon replacing $\boldsymbol{F}_{\mathrm{RF}} \boldsymbol{f}_{\mathrm{BB}}^{d}[m]$ in (15) by $\boldsymbol{f}_{\mathrm{ZF}}^{d}[m]$, the DL power allocation problem can now be formulated as

$$
\begin{gathered}
\max _{p_{m, d}} \sum_{d=1}^{D} \sum_{m \in \mathcal{M}_{d}} \log _{2}\left(1+p_{m, d} H_{m, d}\right) \\
\text { s.t. }(13 g),(15 b)
\end{gathered}
$$

where

$$
H_{m, d}=\frac{\left|\frac{\boldsymbol{h}_{d}^{H}[m] \boldsymbol{f}_{\mathrm{FF}}^{d}[m]}{\left\|\boldsymbol{f}_{\mathrm{ZF}}^{d}\right\|_{2}}\right|^{2}}{\left(\sum_{i \in \mathcal{D}_{m}, i \neq d} p_{m, i}\left|\frac{\boldsymbol{h}_{d}^{H}[m] \boldsymbol{f}_{\mathrm{ZF}}^{i}[m]}{\left\|\boldsymbol{f}_{\mathrm{ZF}}^{i}[m]\right\|_{2}}\right|^{2}+\sigma^{2}\right)}
$$

It can be readily shown that this is a convex optimization problem $^{6}$. Therefore, by introducing the Lagrange multiplier, the equivalent cost function can be formulated as

$$
\begin{aligned}
L & =\sum_{d=1}^{D} \sum_{m \in \mathcal{M}_{d}} \log _{2}\left(1+p_{m, d} H_{m, d}\right) \\
& +\lambda_{1}\left(\sum_{d=1}^{D} \sum_{m \in \mathcal{M}_{d}} p_{m, d}-P_{\mathrm{DL}}\right) \\
& +\sum_{d=2}^{D} \lambda_{d}\left(\sum_{m \in \mathcal{M}_{1}} \log _{2}\left(1+p_{m, 1} H_{m, 1}\right)\right. \\
& \left.-\frac{\gamma_{1}}{\gamma_{d}} \sum_{m \in \mathcal{M}_{d}} \log _{2}\left(1+p_{m, d} H_{m, d}\right)\right)
\end{aligned}
$$

In order to obtain the optimal $\left\{p_{m, d}\right\}$ from (20), by following [33], the power allocation can be divided into two parts, namely the power allocation among the subcarriers of a DL MS for given power $P_{d}$ of this MS and the power allocation among the DL MSs. Specifically, when allocating the power $P_{d}$ of the $d$-th DL MS to its $\left|\mathcal{M}_{d}\right|$ subcarriers, we assume without any loss of generality that $H_{1, d} \leq H_{2, d} \leq \ldots \leq H_{\left|\mathcal{M}_{d}\right|, d}$. Then, it can be shown that with the aid of the KKT conditions [34], we have

$$
\begin{aligned}
& p_{m, d}=p_{1, d}+\frac{H_{m, d}-H_{1, d}}{H_{m, d} H_{1, d}}, \text { and } \\
& P_{d}=\left|\mathcal{M}_{d}\right| p_{1, d}+\sum_{m=2}^{\left|\mathcal{M}_{d}\right|} \frac{H_{m, d}-H_{1, d}}{H_{m, d} H_{1, d}}
\end{aligned}
$$

for $m \in \mathcal{M}_{d}$ and $d=1,2, \ldots, D$. The power allocation of (21) implies that the optimal power assignment among the different subcarriers of a DL MS follows the water-filling principles [35]. Hence, it may happen that some subcarriers of a DL MS may be allocated negative power. If this happens, those subcarriers with negative power are removed from the subcarrier set of the MS.

In the context of the power allocation among different DL MSs, the solutions can be obtained via solving the two sets

${ }^{6}$ Note that, as the result of ZF precoding, the interference imposed by the other MSs on the $d$-th DL MS is approximately zero, i.e., we have $\sum_{i \in \mathcal{D}_{m}, i \neq d}\left|\frac{\boldsymbol{h}_{d}^{H}[m] \boldsymbol{f}_{\mathrm{ZF}}^{i}[m]}{\left\|\boldsymbol{f}_{\mathrm{ZF}}^{i}[m]\right\|_{2}}\right|^{2} \approx 0$. Therefore, $H_{m, d}$ is irrelevant to the variables $\left\{p_{m, i}\right\}_{i \neq d}$. of nonlinear equations by the Newton-Raphson method [33], expressed as,

$$
\begin{aligned}
& \frac{\gamma_{1}}{\gamma_{d}}=\frac{\left|\mathcal{M}_{1}\right|\left(\log _{2}\left(1+H_{1,1} \frac{P_{1}-V_{1}}{\left|\mathcal{M}_{1}\right|}\right)+\log _{2} G_{1}\right)}{\left|\mathcal{M}_{d}\right|\left(\log _{2}\left(1+H_{1, d} \frac{P_{d}-V_{d}}{\left|\mathcal{M}_{d}\right|}\right)+\log _{2} G_{d}\right)}, \text { and } \\
& \sum_{d=1}^{D} P_{d}=P_{\mathrm{DL}}
\end{aligned}
$$

for $d=1,2, . ., D$, where $V_{d}=\sum_{m=2}^{\left|\mathcal{M}_{d}\right|} \frac{H_{m, d}-H_{1, d}}{H_{m, d} H_{1, d}}$ and $G_{d}=$ $\left(\prod_{m=2}^{\left|\mathcal{M}_{d}\right|} \frac{H_{m, d}}{H_{1, d}}\right)^{\frac{1}{\left|\mathcal{M}_{d}\right|}}$. To summarize, the power allocation of DL is stated in Algorithm 2.

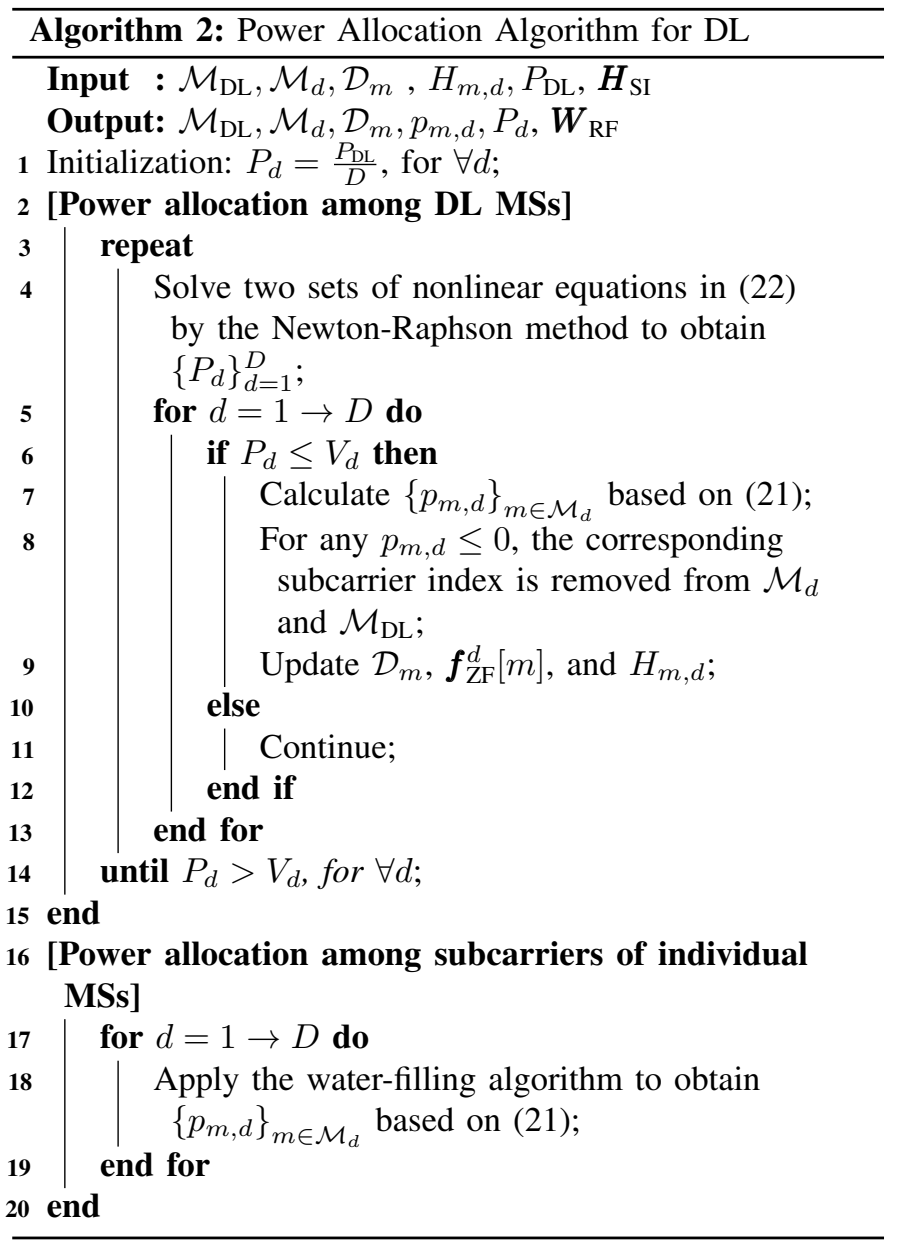

2) Uplink Power Allocation: For UL, power allocation is only among the subcarriers of a single UL MS. As there is no fairness issue, the objective during this stage is solely to maximize data rate. Hence, the water-filling power allocation can be executed based on the similar equation in (21) but under the constraint of the total power of an individual MS.

\section{Hybrid BEAMFORMER DESIGN ASSOCIATED WITH RESOURCE ALLOCATION}

In this section, we first propose two approaches to design the hybrid precoder associated with RA. Then, an adaptive 
SIC algorithm is introduced for practical FD systems via the hybrid combiner design.

\section{A. Hybrid Precoder}

1) Factorization Approach: In order to obtain $\boldsymbol{F}_{\mathrm{RF}}$ and $f_{\mathrm{BB}}^{d}[m], m \in \mathcal{M}_{\mathrm{DL}}, d \in \mathcal{D}_{m}$, the factorization approach for designing the hybrid precoder from $\boldsymbol{f}_{\mathrm{ZF}}^{d}[m]$ can be formulated as

$$
\min _{\boldsymbol{F}_{\mathrm{RF}},\left\{\boldsymbol{f}_{\mathrm{BB}}^{d}[m]\right\}} \sum_{\substack{m \in \mathcal{M}_{\mathrm{DL}} \\ \text { s.t. }}} \sum_{d \in \mathcal{D}_{m}}\left\|\boldsymbol{f}_{\mathrm{ZF}}^{d}[m]-\boldsymbol{F}_{\mathrm{RF}} \boldsymbol{f}_{\mathrm{BB}}^{d}[m]\right\|_{2}^{2}
$$

It is noteworthy that our factorization approach differs from the one considered in [25] and [36], in which the power constraint is set to $\|\left.\boldsymbol{F}_{\mathrm{RF}} \boldsymbol{F}_{\mathrm{BB}}[m]\right|_{F^{2}} ^{2}=\left|\mathcal{D}_{m}\right|$ with $\boldsymbol{F}_{\mathrm{BB}}[m]=\left[\boldsymbol{f}_{\mathrm{BB}}^{1}[m], \boldsymbol{f}_{\mathrm{BB}}^{2}[m], \ldots, \boldsymbol{f}_{\mathrm{BB}}^{\left|\mathcal{D}_{m}\right|}[m]\right]$. In our optimization, as shown in (23), the more strict constraints of $\left\|\boldsymbol{F}_{\mathrm{RF}} \boldsymbol{f}_{\mathrm{BB}}^{d}[m]\right\|_{2}^{2}=1, \forall m, d$ are imposed. In order to solve the problem of (23), first, we minimize $\left\|\boldsymbol{f}_{\mathrm{ZF}}^{d}[m]-\boldsymbol{F}_{\mathrm{RF}} \boldsymbol{f}_{\mathrm{BB}}^{d}[m]\right\|_{2}^{2}$ for a given $\boldsymbol{F}_{\mathrm{RF}}$. This gives a least square (LS) solution to $\left\{\boldsymbol{f}_{d}[m]\right\}$, i.e., $\hat{\boldsymbol{f}}_{\mathrm{BB}}^{d}[m]=\boldsymbol{F}_{\mathrm{RF}}^{\dagger} \boldsymbol{f}_{\mathrm{ZF}}^{d}[m]$, where $\boldsymbol{F}_{\mathrm{RF}}^{\dagger}=$ $\left(\boldsymbol{F}_{\mathrm{RF}}^{H} \boldsymbol{F}_{\mathrm{RF}}\right)^{-1} \boldsymbol{F}_{\mathrm{RF}}^{H}$, as $N>N_{\mathrm{RF}}$ and $\boldsymbol{F}_{\mathrm{RF}}$ is assumed to be a full column rank matrix. After the power normalization to meet $\left\|\boldsymbol{F}_{\mathrm{RF}} \boldsymbol{f}_{\mathrm{BB}}^{d}[m]\right\|_{2}^{2}=1$, we obtain

$$
\tilde{\boldsymbol{f}}_{\mathrm{BB}}^{d}[m]=\frac{\hat{\boldsymbol{f}}_{\mathrm{BB}}^{d}[m]}{\| \boldsymbol{F}_{\mathrm{RF}}^{\hat{\boldsymbol{f}}_{\mathrm{BB}}^{d}[m] \|_{2}}}
$$

Then, substituting $\tilde{\boldsymbol{f}}_{\mathrm{BB}}^{d}[m]$ for $\boldsymbol{f}_{\mathrm{BB}}^{d}[m]$ into (23), the cost function of the problem can be rewritten as

$$
\Phi\left(\boldsymbol{F}_{\mathrm{RF}}\right)=\sum_{m \in \mathcal{M}_{\mathrm{DL}}} \sum_{d \in \mathcal{D}_{m}}\left\|\boldsymbol{f}_{\mathrm{ZF}}^{d}[m]-\boldsymbol{F}_{\mathrm{RF}} \tilde{\boldsymbol{f}}_{\mathrm{BB}}^{d}[m]\right\|_{2}^{2}
$$

According to the Projected Gradient Descent (PGD) principle [37], the local minimum can be reached via updating $F_{\mathrm{RF}}$ along the negative conjugate gradient direction and then, projecting the solution to the feasible set where the elements of matrix have unit modulus. Hence, after obtaining the conjugate gradient as shown in Appendix A, the above-mentioned two steps can be expressed as

$$
\begin{aligned}
& \boldsymbol{F}_{\mathrm{RF}}^{(i+1)}=\boldsymbol{F}_{\mathrm{RF}}^{(i)}-l \frac{\partial(\Phi)}{\partial \boldsymbol{F}_{\mathrm{RF}}^{*(i)}}, \\
& \boldsymbol{F}_{\mathrm{RF}}^{(i+1)}=\arg \left(\boldsymbol{F}_{\mathrm{RF}}^{(i+1)}\right)
\end{aligned}
$$

where $l$ denotes a positive decreasing step size towards the local optimum. Once the optimal $\boldsymbol{F}_{\mathrm{RF}}$ is obtained, $\boldsymbol{f}_{\mathrm{BB}}^{d}[m]$ can be derived as

$$
\boldsymbol{f}_{\mathrm{BB}}^{d}[m]=\left(\boldsymbol{F}_{\mathrm{RF}}^{(i)}\right)^{\dagger} \boldsymbol{f}_{\mathrm{ZF}}^{d}[m] /\left\|\boldsymbol{F}_{\mathrm{RF}}^{(i)}\left(\boldsymbol{F}_{\mathrm{RF}}^{(i)}\right)^{\dagger} \boldsymbol{f}_{\mathrm{ZF}}^{d}[m]\right\|_{2}, \forall d, m
$$

2) Direct Approach: Although the PGD algorithm with a decreasing step size guarantees the convergence to a local minimum, the difference between $\boldsymbol{f}_{\mathrm{ZF}}^{d}[m]$ and $\boldsymbol{F}_{\mathrm{RF}} \boldsymbol{f}_{d}[m]$ may be increased by RA, especially when the number of RF chains is small, which results in that the interference in (19) from other MSs cannot be approximated to zero. In this case, Algorithm 2 may be hard to achieve the proportional rates among DL MSs at a sufficient accuracy, as the result that $H_{m, d}$ in (20) also includes $p_{m, i}, i \neq d$, in addition to the desired $p_{m, d}$. Consequently, due to the deviation introduced by the hybrid precoder, the problem of (18) is no longer a convex optimization problem, which is hence prohibitive from being solved by an efficient approach. Therefore, for comparison purposes, below we present a so-called Direct Approach (DA) to derive $\boldsymbol{F}_{\mathrm{RF}}$ and $\boldsymbol{f}_{d}[\mathrm{~m}]$, which is capable of providing the precise proportions of fairness.

To begin with, according to [38], suboptimal $\boldsymbol{F}_{\mathrm{RF}}$ can be obtained by solving the following optimization problem :

$$
\begin{aligned}
& \max _{\boldsymbol{F}_{\mathrm{RF}}} \log _{2}\left|\boldsymbol{I}+\frac{P_{\mathrm{DL}}}{\sigma^{2} N N_{\mathrm{RF}}} \boldsymbol{F}_{\mathrm{RF}}^{H} \tilde{\boldsymbol{H}} \boldsymbol{F}_{\mathrm{RF}}\right| \\
& \text { s.t. }(13 j)
\end{aligned}
$$

where $\tilde{\boldsymbol{H}}=\frac{1}{\left|\mathcal{M}_{\mathrm{DL}}\right|} \sum_{m \in \mathcal{M}_{\mathrm{DL}}}\left(\boldsymbol{H}_{\mathrm{DL}}[m]^{H} \boldsymbol{H}_{\mathrm{DL}}[m]\right)$. Next, the digital $\mathrm{ZF}$ precoders $\boldsymbol{F}_{\mathrm{ZF}}^{\mathrm{BB}}[m]$ are obtained by replacing $\boldsymbol{H}_{\mathrm{DL}}[m]$ with $\boldsymbol{H}_{\text {eq }}[m]$ in (17), where $\boldsymbol{H}_{\mathrm{eq}}[m]=\boldsymbol{H}_{\mathrm{DL}}[m] \boldsymbol{F}_{\mathrm{RF}}$. Then, after normalization, we can solve the optimization problem by letting $\boldsymbol{F}_{\mathrm{ZF}}[m]$ in (17) equal to $\boldsymbol{F}_{\mathrm{RF}} \boldsymbol{F}_{\mathrm{ZF}}^{\mathrm{BB}}[m]$. Since the optimization (18) is convex, which enables us to derive the solutions directly using Algorithm 2, which yields the precise proportions of fairness. The details can be found in Algorithm 3 as shown below.

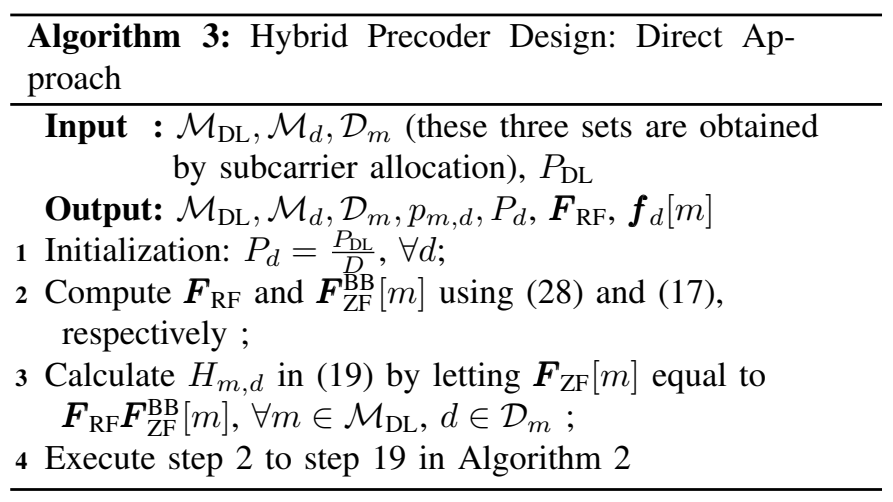

It is noteworthy that both the proposed hybrid beamforming algorithms have the same overall computation complexity, expressed as $\mathcal{O}\left(N N_{\mathrm{RF}}^{2}\right)$. However, it can be observed from Algorithm 3 that the DA method is coupled with power allocation. This means that the hybrid precoders in the DA method need to be continuously updated during power allocation, as shown in Algorithm 2 (line 9), which leads to an increased complexity. By contrast, the hybrid beamformers in the PGD method are independent of the power allocation, which are hence simpler for practical implementation. 


\section{B. Design of Hybrid Combiner with SI Suppression}

During resource allocation, the effect of SI is ignored by assuming perfect SI suppression. However, existing SIC methods are unable to provide sufficient SI reduction while keeping a low complexity and overhead at the same time. Hence in this section, we introduce an adaptive beamforming based SIC algorithm by exploiting the large number of antennas employed in mmWave systems.

First, according to (11), the total power of the SI signal $\boldsymbol{r}_{\mathrm{SI}}$ after analog combining can be expressed as

$$
\begin{aligned}
& \operatorname{Tr}\left(\operatorname{Cov}\left(\boldsymbol{r}_{\mathrm{SI}}\right)\right)=\xi \operatorname{Tr}\left(\boldsymbol{F}_{\mathrm{RF}}^{H} \boldsymbol{H}_{\mathrm{SI}}^{H} \boldsymbol{W}_{\mathrm{RF}} \boldsymbol{W}_{\mathrm{RF}}^{H} \boldsymbol{H}_{\mathrm{SI}} \boldsymbol{F}_{\mathrm{RF}} \times\right. \\
& \left.\left(\sum_{d=1}^{D} \sum_{m \in \mathcal{M}_{d}} p_{m, d} \boldsymbol{f}_{\mathrm{BB}}^{d}[m] \boldsymbol{f}_{\mathrm{BB}}^{d, H}[m]\right)\right)
\end{aligned}
$$

from which we can observe that the SI power is proportional to $\left\|\boldsymbol{W}_{\mathrm{RF}}^{H} \boldsymbol{H}_{\mathrm{SI}} \boldsymbol{F}_{\mathrm{RF}}\right\|_{F}^{2}$. Since $\boldsymbol{F}_{\mathrm{RF}}$ has already been determined by DL power allocation and is fixed, the suppression of SI can only be depended on the design of the analog combiner $W_{\mathrm{RF}}$. Therefore, we have the optimization problem stated as

$$
\begin{aligned}
& \min _{\boldsymbol{W}_{\mathrm{RF}}}\left\|\boldsymbol{W}_{\mathrm{RF}}^{H} \boldsymbol{H}_{\mathrm{SI}} \boldsymbol{F}_{\mathrm{RF}}\right\|_{F}^{2} \\
& \text { s.t. }(13 j)
\end{aligned}
$$

The problem (30) can be solved by the cyclic coordinate descent (CCD) algorithm via iteratively optimizing the individual elements of $W_{\mathrm{RF}}$, the details of which can be found in [7] and are not repeated for brevity. After obtaining the analog combiner for SIC, the optimal digital combiner for a subcarrier can be derived based on conventional MMSE approach [39].

Note that our proposed SIC is operated independently from RA. Hence, the process of SIC does not have any impact on the operations of RA, which were considered in the previous sections.

\section{PERformance Results}

In this section, we firstly present the simulation results for the MDD/MU-MIMO mmWave systems with the proposed RA algorithms, when the QoS requirements of both UL and DL MSs are imposed. Then, to tackle the problem of SI in MDD RA systems, the performance of our proposed SI reduction method is investigated in Section VI-B. All results are obtained from the MATLAB-based simulations.

\section{A. Resource Allocation in MDD/MU-MIMO mmWave Systems}

First, let us demonstrate the performance of the MDD/MUMIMO mmWave systems with our proposed RA algorithms. To begin with, we show the performance upper-bound by following [27] without considering the fairness constraints, when subcarrier allocation is achieved using the UG algorithm, while transmit power is assigned by the water-filling algorithm. However, we should note that in [27], all MSs and BS are equipped with single antenna and are operated in HD mode. Hence, to make it compatible with our MDD/MU-MIMO system, we consider a modified UG (MUG) algorithm for the scheme of [27] to operate the second and third stages of subcarrier allocation but keep the water-filling algorithm for power allocation. In the MUG algorithm, we assume that interuser interference is mitigated by a ZF precoder. Then, each subcarrier may be assigned to multiple MSs with the best channel gains under the constraint of $\left|\mathcal{D}_{m}\right| \leq N_{\mathrm{RF}}$. Afterwards, $\mathrm{BS}$ allocates the transmit power to all $\mathrm{DL}$ subcarriers based on the water-filling principle, while UL power assignment is implemented via water-filling for each UL MS's subcarriers. Additionally, we also compare another RA method, namely MUG-PowAve. With the MUG-PowAve, subcarrier allocation is the same as the MUG algorithm, but DL power is firstly evenly distributed to all DL subcarriers, and then water-filling is used to assign the power of a subcarrier to the MSs sharing this subcarrier. Note that this RA approach has been considered in many references, such as, in [25,39].

In this subsection, we consider a MDD/MU-MIMO mmWave system operated in $28 \mathrm{GHz}$ with the SIC capability of $\xi=-100 \mathrm{~dB}$, where BS employs $N=32$ transmit antennas and $N_{\mathrm{RF}}=16 \mathrm{DL} \mathrm{RF}$ chains to support $D=20$ DL MSs, and $\bar{N}=128$ receive antennas and $\bar{N}_{\mathrm{RF}}=16$ UL RF chains to serve $\bar{D}=10$ UL MSs. All MSs are randomly distributed with their distances from BS being between $50 m$ to $200 m$, i.e., $d_{m} \in[50,200]$. The pathloss is $P_{L}(\mathrm{~dB})=72+29.2 \cdot \log 10\left(d_{m}\right)$. The total number of subcarriers are $M=64$, in which the number of DL and UL subcarriers used for transmissions satisfy $\left|\mathcal{M}_{\mathrm{DL}}\right|=$ $\left|\mathcal{M}_{\mathrm{UL}}\right| \leq \frac{M}{2}=32$. The transmit power of BS transmitter is $P_{\mathrm{DL}}=12 \mathrm{~W}$, and all UL MSs have the same transmit power of $P_{\bar{d}}=P_{\mathrm{DL}} / D=0.6 \mathrm{~W}$. All the above-mentioned parameter values are default values, unless they are stated specifically. The default proportional fairness among DL and UL MSs are $\left\{\gamma_{i}=1\right\}_{i=1}^{D}$ and $\left\{\eta_{i}=1\right\}_{i=1}^{\bar{D}}$, respectively. Additionally, we will consider the other two fairness constraints of $\gamma_{1}=5$ and $\gamma_{1}=10$, respectively, while the other values remain the same as the default value of 1 . Besides, we assume that the transmit and receive arrays at BS are the ULA arrays with the half-wavelength spacing between two adjacent antenna elements. Both DL and UL channels are assumed to have $T=6$ delay taps and $L_{d}=4$ paths. The channel gains obey the distribution $v_{l, d} \in \mathcal{C N}(0,1)$, and the AoA/AoD azimuth angles are assumed to be uniformly distributed in $[0,2 \pi]$. Furthermore, the noise variance $\sigma^{2}$ is set to $10^{-13} \mathrm{~W}$. The results are obtained via average of 100 channel realizations.

Firstly in Fig. 4, we demonstrate the performance of the MDD/MU-MIMO mmWave systems with our proposed RA algorithm having different fairness constraints. Here, it is assumed that full-digital precoders are employed at BS transmitter. Fig. 4 demonstrates that the proposed RA with $\left\{\gamma_{i}=1\right\}_{i=1}^{D}$ slightly outperforms the MUG-PowAve method but slightly worse than the MUG method in terms of the sum rate per MS. As $\gamma_{1}$ increases, the average sum rate per MS achieved by the proposed RA becomes lower. The reason behind is that all MSs in the system experience largescale fading. Due to the high level path-loss in mmWave communications, if one MS far away from the BS imposes the highest demand for a data rate, which corresponds to $\gamma_{1}=5$ or 10 , the BS has to assign most of the transmit power to 


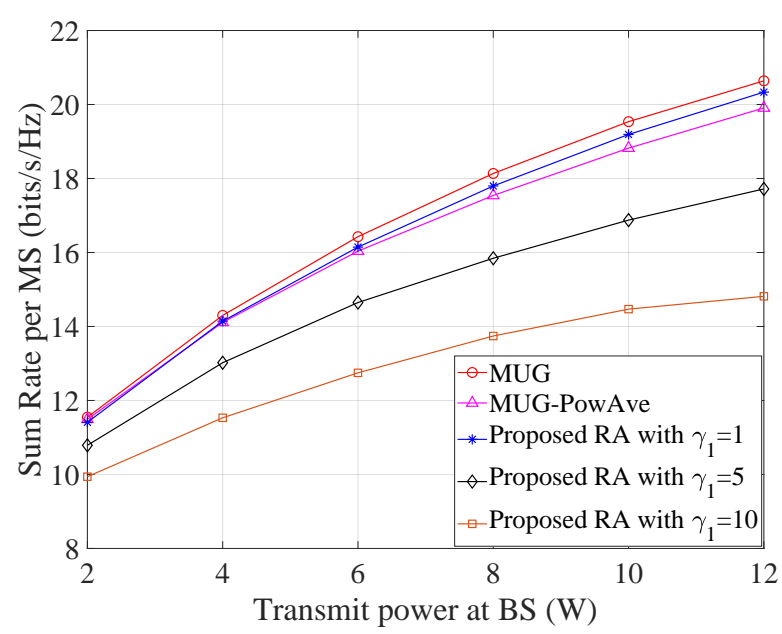

Fig. 4. Performance comparison of the MUG algorithm and the proposed algorithm with different fairness constraints, when $\bar{N}=128 \bar{N}_{\mathrm{RF}}=16$, $N=32, N_{\mathrm{RF}}=16, D=20, \bar{D}=10$.

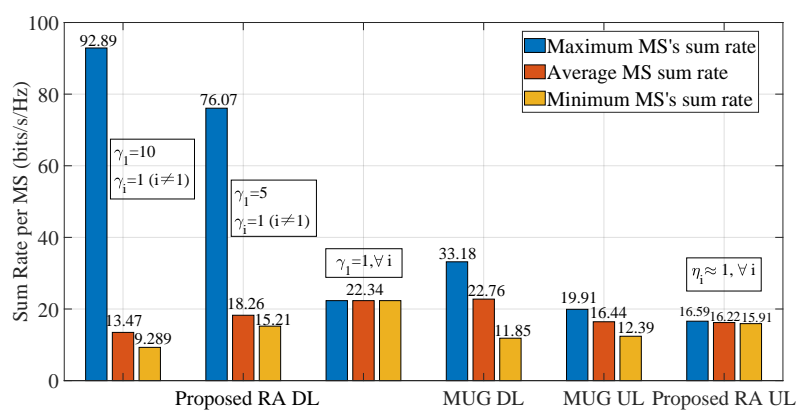

Fig. 5. Fairness comparison of MUG algorithm and proposed RA algorithm, when $P_{\mathrm{DL}}=12 W, \bar{N}=128 \bar{N}_{\mathrm{RF}}=16, N=32, N_{\mathrm{RF}}=16, D=20$, $\bar{D}=10$.

this MS, making the power allocation inefficient and hence the degradation of overall data rate.

Fig. 5 shows the fairness comparison between the MUG algorithm and the proposed RA algorithm in different cases. Explicitly, our proposed RA method can achieve the nearaccurate proportions of rates for the DL MSs, when $\gamma_{1}$ has different values. Fig. 5 also shows the corresponding results of the MUG algorithm, when $\left\{\gamma_{i}=1\right\}_{i=1}^{D}$ and $\left\{\eta_{i}=1\right\}_{i=1}^{\bar{D}}$. We can see that the MUG algorithm slightly outperforms the proposed RA algorithm in terms of the average sum rate per DL or UL MS. However, it causes a big difference between the maximum and minimum rates (about $21 \mathrm{bits} / \mathrm{s} / \mathrm{Hz}$ ), which explains the possible poor performance attained by the MSs located at cell edge. By contrast, for the proposed RA algorithm, the proportional fairness of all DL MSs can be guaranteed. However, as the fairness in UL case is only considered via subcarrier allocation, the achieved rates of UL MSs have small fluctuation. Nevertheless, they are still much more stable than that obtained by the MUG algorithm.

Next, we compare the complexity of our proposed and the competing RA methods. In all these schemes, RA is divided into two stages, subcarrier allocation and power allocation. Both MUG and MUG-PowAve algorithms use the same
TABLE I

COMPLEXITY COMPARISON OF THE PROPOSED AND COMPETING RA METHODS IN MDD SYSTEMS

\begin{tabular}{|c|c|c|c|}
\hline Method & Complexity & Sum Rate & Feature \\
\hline MUG & $\mathcal{O}(2 M)$ & High & Optimal RA method \\
\hline MUG-PowAve & $\mathcal{O}\left(N_{\mathrm{RF}} M+M\right)$ & Medium & Easy-to-implement \\
\hline Proposed $\left(\gamma_{1}=1\right)$ & $\mathcal{O}\left(D_{\text {sum }} M+D^{3}\right)$ & High & Fairness guaranteed \\
\hline
\end{tabular}

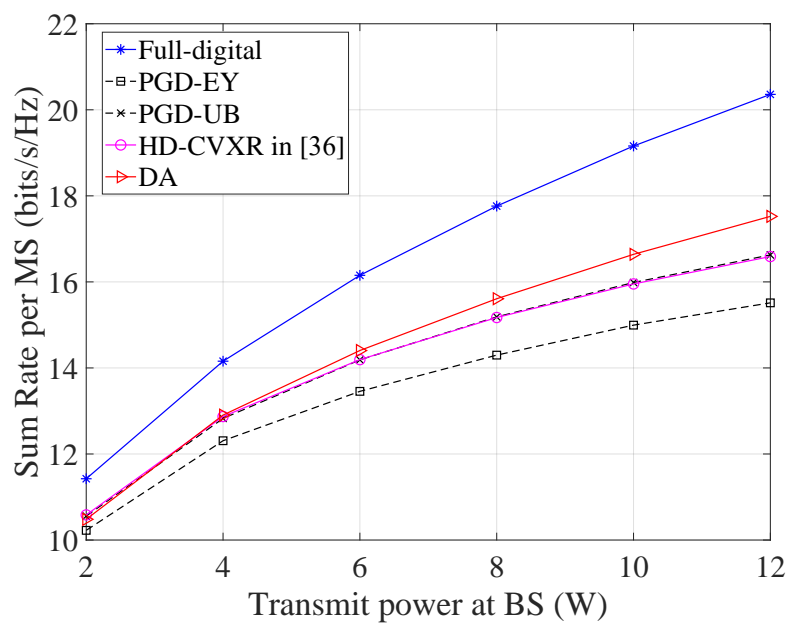

Fig. 6. Sum rate versus transmit power of BS when different hybrid precoders are employed and when assuming $\bar{N}=128, \bar{N}_{\mathrm{RF}}=16, N=32, N_{\mathrm{RF}}=16$, $D=20, \bar{D}=10,\left\{\gamma_{i}=1\right\}_{i=1}^{D}$ and $\left\{\eta_{i}=1\right\}_{i=1}^{\bar{D}}$.

subcarrier allocation method, which has the complexity of $\mathcal{O}(M)$, while the proposed method needs to meet the fairness constraint and has the complexity of $\mathcal{O}\left(D_{\text {sum }} M\right)$. For power allocation, both MUG and MUG-PowAve apply the waterfilling algorithm, whose complexity is linearly proportional to the number of variables used in the optimization problem [40]. Therefore, these two methods have the complexity of $\mathcal{O}(M)$ and $\mathcal{O}\left(N_{\mathrm{RF}} M\right)$, respectively. By contrast, the proposed algorithm needs to solve the nonlinear equations and calculate the matrix inversion when applying the New-Raphson method. These lead to a complexity of $\mathcal{O}\left(D^{3}\right)$. Overall, the computational complexity of the considered RA algorithms is summarized in Table I. In a nutshell, although our proposed algorithm has higher complexity than the other two UG algorithms, when the number of MSs becomes large, this extra complexity investment lead to the promising performance and at the same time, the near-accurate fairness defined for different MSs.

Fig. 6 and 7 show the sum rate performance of the MDD/MU-MIMO mmWave systems, when various hybrid precoders are employed. Here PGD-EY indicates the hybrid precoder with $\boldsymbol{F}_{\mathrm{RF}}$ initialized by the Eckart-Young theorem [25]. PGD-UB indicates the hybrid precoder with $\boldsymbol{F}_{\mathrm{RF}}$ initialized by maximizing the upper bound of DL's spectral efficiency for a given $\boldsymbol{F}_{\mathrm{BB}}$ [39]. Additionally, the hybrid precoder design by convex relaxation (HD-CVXR) presented in [36] is compared, which is demonstrated to provide a high spectral efficiency in the conventional hybrid beamforming systems. Fig. 6 shows that when $N_{\mathrm{RF}}=16$, DA is capable of achieving the highest sum rate among the considered schemes, 


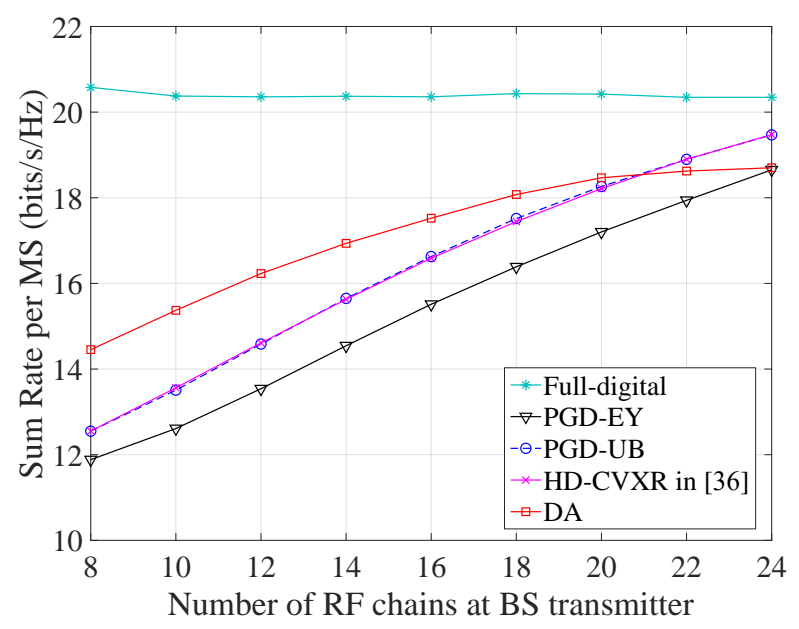

Fig. 7. Sum rate versus the number of RF chains at BS transmitter, when different hybrid precoder designs are employed and when assuming $P_{\mathrm{DL}}=$ $12 W, \bar{N}=128, \bar{N}_{\mathrm{RF}}=16, N=32, D=20, \bar{D}=10,\left\{\gamma_{i}=1\right\}_{i=1}^{D}$ and $\left\{\eta_{i}=1\right\}_{i=1}^{\bar{D}}$.

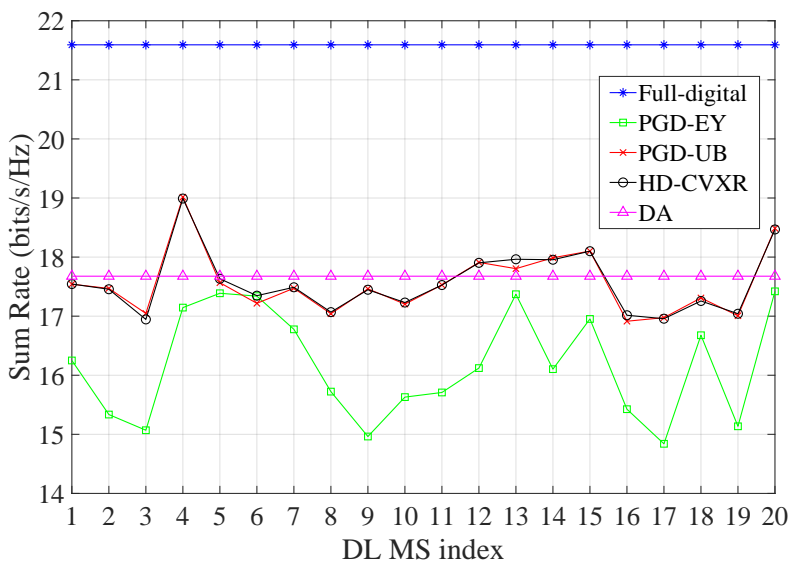

Fig. 8. Rates of individual DL MSs achieved for one channel realization, when different hybrid precoders are employed and when assuming $P_{\mathrm{DL}}=12 \mathrm{~W}$ $\bar{N}=128, \bar{N}_{\mathrm{RF}}=16, N=32, N_{\mathrm{RF}}=20, D=20$ and $\left\{\gamma_{i}=1\right\}_{i=1}^{D}$.

which is only $3 \mathrm{bits} / \mathrm{s} / \mathrm{Hz}$ lower than that obtained by the fulldigital $\mathrm{ZF}$ solution. The reason behind is that the factorization of the full-digital ZF precoder using the approach, such as PGD and HD-CVXR, introduces inter-user interference within a subcarrier. This interference is further amplified by power allocation. The merit of DA is that the inter-user interference can be efficiently mitigated by applying $\mathrm{ZF}$ processing for $\boldsymbol{F}_{\mathrm{BB}}$. As shown in Fig. 6, the initialization of RF precoder by the PGD method generates a big impact on the sum rate performance, and the one from [39] is better for our system than the other one from [25].

From Fig. 7, we observe that both the PGD-UB and HDCVXR algorithms surpass the DA, when the number of RF chains is big, which converge to the upper bound performance achieved by the full-digital ZF. This can be explained as follows. When the number of RF chains is relatively big, the difference between the full-digital $\mathrm{ZF}$ and the hybrid precoders becomes sufficiently small, making the inter-user interference yields little influence on the sum rate even after

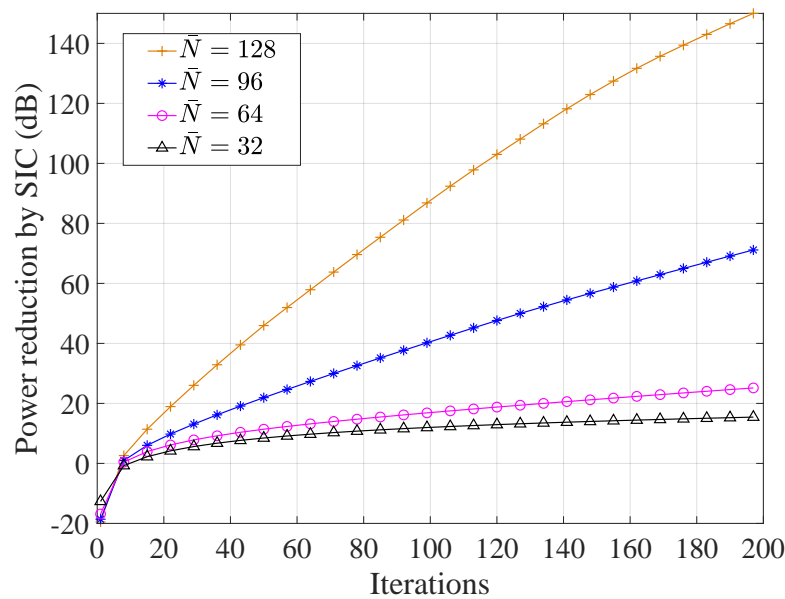

Fig. 9. SIC performance with respect to different number of antenna elements at receiver, when assuming $P_{\mathrm{DL}}=12 W, \bar{N}_{\mathrm{RF}}=16, N=32, N_{\mathrm{RF}}=16$, $\varphi=120^{\circ}, \kappa=10$.

power allocation.

Finally, Fig. 8 illustrates the rates achieved by individual DL MSs for one channel realization. When given the fairness constraint of $\left\{\gamma_{i}=1\right\}_{i=1}^{D}$, Fig. 8 shows that the DA with higher implementation complexity can achieve the exact fairness requirements and the stable rates for all DL MSs via solving the optimization problem in (15) with respect to every channel realization. By contrast, the factorization operation in the full-digital precoder (e.g., PGD and HD-CVXR) introduces inter-user interference, which imposes cost on the equal rate constraint and leads to the fluctuation of individual MSs' DL rates.

\section{B. Self-Interference Cancellation}

In Section VI-A, we assumed the system with ideal SI suppression associated with a SIC parameter $\xi=-100 \mathrm{~dB}$, provided by existing approaches. However, to the best of our knowledge, none existing SIC methods are able to provide 100 $\mathrm{dB}$ SI reduction in hybrid beamforming aided FD systems. Therefore, in this subsection, we assume that there are no other SIC methods available, meaning that $\xi=0 \mathrm{~dB}$ in the model of $\boldsymbol{r}_{\mathrm{SI}}$. Hence, all SI reduction depends on our proposed methods in Section V-B. For performance study, apart from the default values used in Section VI-A, we assume that for the LOS component of SI channel, the distance $r_{i j}$ between the $i$-th element of transmit array and the $j$-th element of receive array is set according to [26], and the default angle between transmit array and receive array is $\varphi=120^{\circ}$. For NLOS components, we assume that the channel has $L_{n}=6$ paths and the complex gain $\alpha_{l}$, AoA and AoD have the same features as the communication channels stated in Section VI-A.

In Fig. 9, we plot the SIC performance versus the number of iterations operated by the proposed SIC algorithm. It is observed that for a given transmit antenna array and a given number of iterations, more SI can be mitigated, when BS is equipped with more receive antenna elements. The reason behind is that as the number of receive antenna elements 


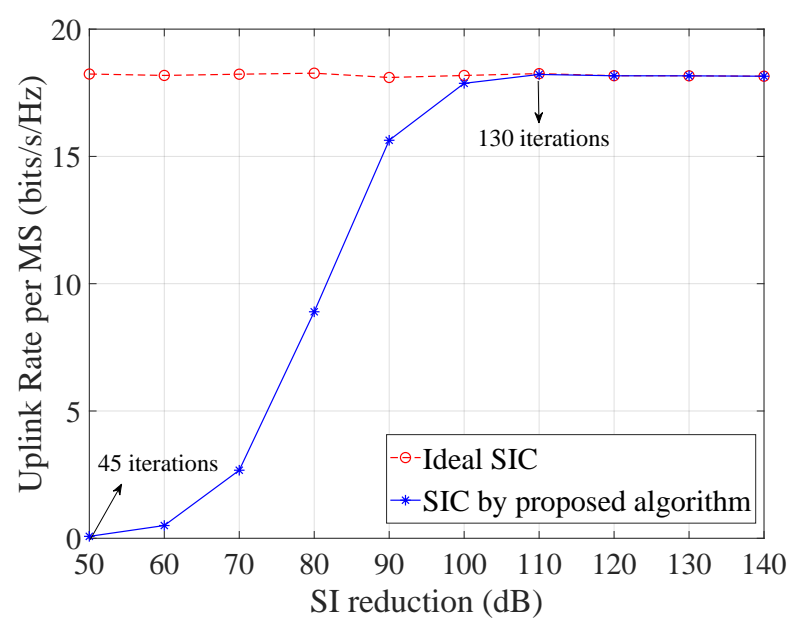

Fig. 10. Uplink performance with ideal SIC and the SIC provided by our proposed algorithm, when assuming that $P_{\mathrm{DL}}=12 \mathrm{~W}, \bar{N}=128, \bar{N}_{\mathrm{RF}}=16$, $N=32, N_{\mathrm{RF}}=16, \varphi=120^{\circ}, \kappa=10$.

increases, the null space of $\boldsymbol{H}_{\mathrm{SI}}^{H}$ has a bigger rank. Therefore, the receiver is more capable of suppressing SI signals.

Fig. 10 studies the influence of the SI suppression provided by our proposed SIC algorithm on the desired UL transmissions, where ideal SI cancellation means that $\boldsymbol{r}_{\mathrm{SI}}=\mathbf{0}$ in (11) during signal detection. From Fig. 10, we can observe that to attain the same performance as the ideal system without SI, a practical system should provide at least $110 \mathrm{~dB}$ SI reduction, which requires our proposed SIC method to operate 130 iterations. The insufficient SI suppression may lead to large quantization noise and make RA inefficient in FD systems. It is worth noting that in practice, the SI channel varies very slowly due to long coherence time, as both transmitter and receiver are fixed at BS. Hence, the SIC operations are only required to be executed once after a relatively long time period. Furthermore, a SIC updating process can be started at a nearly converged state, which allows a significantly shorter time than 130 iterations to reach the required SIC. Therefore, the proposed SIC algorithm is efficient for practical operation.

\section{CONCLUSIONS}

In this paper, we have studied the RA problem in the large-scale MDD/MU-MIMO mmWave systems with the consideration of user fairness, SI suppression and hybrid precoder/combiner design. To demonstrate the advantage of MDD, we have first considered the RA based on the unfair greedy algorithm in MU-SISO systems. The studies show that MDD mode has the potential to outperform HD modes, owing to that the MDD mode enables UL and DL to jointly share the available resource. Then, the suboptimal RA algorithms have been proposed for the MDD/MU-MIMO systems to maximize the sum-rate and simultaneously to meet the fairness requirements among DL and UL MSs. Furthermore, two approaches, namely the matrix factorization approach and DA, have been proposed for designing the hybrid precoder associated with the RA schemes. Our studies reveal that when the number of RF chains is relatively small, the DA outperforms the matrix factorization approach. By contrast, when the number of RF chains is relatively big, the matrix factorization approach is a better option for designing precoders. Furthermore, to address the SI problem in MDD systems, we have modeled the influence of SI and proposed an adaptive beamforming aided SIC algorithm embedded in combiner design. Our simulation results demonstrate that the proposed SIC algorithm enable the RA operations free from SI with appropriate antenna deployment, while imposing no impact on the desired UL transmissions.

\section{APPENDIX A}

Conjugate Gradient COMPUTATION FOR PGD

According to (25), we have

$$
\begin{aligned}
\Phi\left(\boldsymbol{F}_{\mathrm{RF}}\right) & =\sum_{m \in \mathcal{M}_{\mathrm{DL}}} \sum_{d \in \mathcal{D}_{m}}\left\|\boldsymbol{f}_{\mathrm{ZF}}^{d}[m]-\boldsymbol{F}_{\mathrm{RF}} \boldsymbol{f}_{\mathrm{BB}}^{\tilde{d}}[m]\right\|_{2}^{2} \\
& =\sum_{m \in \mathcal{M}_{\mathrm{DL}}} \sum_{d \in \mathcal{D}_{m}}\left(\left\|\boldsymbol{f}_{\mathrm{ZF}}^{d}[m]\right\|_{2}^{2}+1\right. \\
& -\underbrace{\operatorname{Tr}\left(\boldsymbol{f}_{\mathrm{ZF}}^{d}[m] \boldsymbol{f}_{\mathrm{BB}}^{\tilde{d}, H}[m] \boldsymbol{F}_{\mathrm{RF}}^{H}+\boldsymbol{F}_{\mathrm{RF}} \tilde{f}_{\mathrm{BB}}^{\tilde{d}}[m] \boldsymbol{f}_{\mathrm{ZF}}^{d, H}[m]\right)}_{\mathrm{A}})
\end{aligned}
$$

To simplify the second term in the above equation, based on (24), we have

$$
\begin{aligned}
\mathrm{A} & =\frac{2 \operatorname{Tr}\left(\boldsymbol{f}_{\mathrm{ZF}}^{d}[m] \boldsymbol{f}_{\mathrm{ZF}}^{d, H}[m] \boldsymbol{F}_{\mathrm{RF}}\left(\boldsymbol{F}_{\mathrm{RF}}^{H} \boldsymbol{F}_{\mathrm{RF}}\right)^{-1} \boldsymbol{F}_{\mathrm{RF}}^{H}\right)}{\left\|\boldsymbol{F}_{\mathrm{RF}}\left(\boldsymbol{F}_{\mathrm{RF}}^{H} \boldsymbol{F}_{\mathrm{RF}}\right)^{-1} \boldsymbol{F}_{\mathrm{RF}}^{H} \boldsymbol{f}_{\mathrm{ZF}}^{d}[m]\right\|_{2}} \\
& =2 \sqrt{\operatorname{Tr}\left(\left(\boldsymbol{F}_{\mathrm{RF}}^{H} \boldsymbol{F}_{\mathrm{RF}}\right)^{-1} \boldsymbol{F}_{\mathrm{RF}}^{H} \boldsymbol{f}_{\mathrm{ZF}}^{d}[m] \boldsymbol{f}_{\mathrm{ZF}}^{d, H}[m] \boldsymbol{F}_{\mathrm{RF}}\right)} \\
& =2\left\|\boldsymbol{b}_{d}[m]\right\|_{2}
\end{aligned}
$$

where $\boldsymbol{b}_{d}[m]=\left(\boldsymbol{F}_{\mathrm{RF}}^{H} \boldsymbol{F}_{\mathrm{RF}}\right)^{-1 / 2} \boldsymbol{F}_{\mathrm{RF}}^{H} \boldsymbol{f}_{\mathrm{ZF}}^{d}[m]$. Hence, the cost function of (25) can be expressed as

$$
\Phi\left(\boldsymbol{F}_{\mathrm{RF}}\right)=\sum_{m \in \mathcal{M}_{\mathrm{DL}}} \sum_{d \in \mathcal{D}_{m}}\left\|\boldsymbol{f}_{\mathrm{ZF}}^{d}[m]\right\|_{2}^{2}-2\left\|\boldsymbol{b}_{d}[m]\right\|_{2}+1
$$

Upon taking the derivative of the (33) with respected to $F_{\mathrm{RF}}^{*}$, we obtain

$$
\begin{array}{r}
\frac{\partial(\Phi)}{\partial \boldsymbol{F}_{\mathrm{RF}}^{*}}=\sum_{m \in \mathcal{M}_{\mathrm{DL}}} \sum_{d \in \mathcal{D}_{m}} \frac{1}{\left\|\boldsymbol{b}_{d}[m]\right\|_{2}}\left(\boldsymbol{F}_{\mathrm{RF}} \boldsymbol{F}_{\mathrm{RF}}^{H} \boldsymbol{f}_{\mathrm{ZF}}^{d}[m] \boldsymbol{f}_{\mathrm{ZF}}^{d, H}[m] \times\right. \\
\left.\boldsymbol{F}_{\mathrm{RF}}\left(\boldsymbol{F}_{\mathrm{RF}}^{H} \boldsymbol{F}_{\mathrm{RF}}\right)^{-2}-\boldsymbol{f}_{\mathrm{ZF}}^{d}[m] \boldsymbol{f}_{\mathrm{ZF}}^{d, H}[m] \boldsymbol{F}_{\mathrm{RF}}\left(\boldsymbol{F}_{\mathrm{RF}}^{H} \boldsymbol{F}_{\mathrm{RF}}\right)^{-1}\right)
\end{array}
$$

\section{REFERENCES}

[1] S. Rangan, T. S. Rappaport, and E. Erkip, "Millimeter wave cellular wireless networks: Potentials and challenges," arXiv preprint arXiv:1401.2560, 2014.

[2] K. E. Kolodziej, B. T. Perry, and J. S. Herd, "In-band full-duplex technology: Techniques and systems survey," IEEE Transactions on Microwave Theory and Techniques, vol. 67, no. 7, pp. 3025-3041, 2019.

[3] Z. Xiao, P. Xia, and X.-G. Xia, "Full-duplex millimeter-wave communication," IEEE Wireless Communications, vol. 24, no. 6, pp. 136-143, 2017.

[4] R. Rajashekar et al., "Multicarrier division duplex aided millimeter wave communications," IEEE Access, vol. 7, pp. 100 719-100732, 2019. 
5] B. King, J. Xia, and S. Boumaiza, "Digitally assisted RF-analog self interference cancellation for wideband full-duplex radios," IEEE Transactions on Circuits and Systems II: Express Briefs, vol. 65, no. 3, pp. 336-340, 2017.

[6] A. T. Le, X. Huang, Y. J. Guo et al., "Beam-based analog selfinterference cancellation in full-duplex MIMO systems," IEEE Transactions on Wireless Communications, vol. 19, no. 4, pp. 2460-2471, 2020.

[7] B. Li, L.-L. Yang, R. G. Maunder, and S. Sun, "Self-interference cancellation and channel estimation in multicarrier-division duplex systems with hybrid beamforming," IEEE Access, vol. 8, pp. 160653-160669, 2020.

[8] L.-L. Yang, Multicarrier communications. John Wiley \& Sons, 2009.

[9] D. Bharadia, E. McMilin, and S. Katti, "Full duplex radios," in $A C M$ SIGCOMM computer communication review, vol. 43, no. 4. ACM, 2013, pp. 375-386.

[10] W. W. Ho and Y.-C. Liang, "Optimal resource allocation for multiuser mimo-ofdm systems with user rate constraints," IEEE Transactions on Vehicular Technology, vol. 58, no. 3, pp. 1190-1203, 2008.

[11] T. F. Maciel and A. Klein, "On the performance, complexity, and fairness of suboptimal resource allocation for multiuser mimo-ofdma systems,' IEEE Transactions on Vehicular Technology, vol. 59, no. 1, pp. 406-419, 2009.

[12] X. Wu, Z. Ma, X. Chen, F. Labeau, and S. Han, "Energy efficiencyaware joint resource allocation and power allocation in multi-user beamforming," IEEE Transactions on Vehicular Technology, vol. 68 , no. 5, pp. 4824-4833, 2019.

[13] V. N. Ha, D. H. Nguyen, and J.-F. Frigon, "Subchannel allocation and hybrid precoding in millimeter-wave OFDMA systems," IEEE Transactions on Wireless Communications, vol. 17, no. 9, pp. 59005914, 2018.

[14] C. Zhao et al., "Mobile edge computing meets mmwave communications: Joint beamforming and resource allocation for system delay minimization," IEEE Transactions on Wireless Communications, vol. 19 no. 4, pp. 2382-2396, 2020.

[15] I. Ahmed, H. Khammari, and A. Shahid, "Resource allocation for transmit hybrid beamforming in decoupled millimeter wave multiuserMIMO downlink," IEEE Access, vol. 5, pp. 170-182, 2016.

[16] C. Nam, C. Joo, and S. Bahk, "Joint subcarrier assignment and power allocation in full-duplex OFDMA networks," IEEE Transactions on Wireless Communications, vol. 14, no. 6, pp. 3108-3119, 2015.

[17] B. Di et al., "Joint user pairing, subchannel, and power allocation in fullduplex multi-user OFDMA networks," IEEE Transactions on Wireless Communications, vol. 15, no. 12, pp. 8260-8272, 2016.

[18] D. W. K. Ng, Y. Wu, and R. Schober, "Power efficient resource allocation for full-duplex radio distributed antenna networks," IEEE Transactions on Wireless Communications, vol. 15, no. 4, pp. 2896-2911, 2016.

[19] M. T. Kabir and C. Masouros, "Delay-constrained beamforming and resource allocation in full duplex systems," IEEE Transactions on Vehicular Technology, vol. 69, no. 3, pp. 3476-3480, 2020.

[20] B. P. Day, A. R. Margetts, D. W. Bliss, and P. Schniter, "Full-duplex MIMO relaying: Achievable rates under limited dynamic range," IEEE Journal on Selected Areas in Communications, vol. 30, no. 8, pp. 15411553, 2012.

[21] K. E. Kolodziej, J. G. McMichael, and B. T. Perry, "Multitap RF canceller for in-band full-duplex wireless communications," IEEE Transactions on Wireless Communications, vol. 15, no. 6, pp. 4321-4334, 2016.

[22] B. Debaillie et al., "Analog/RF solutions enabling compact full-duplex radios," IEEE Journal on Selected Areas in Communications, vol. 32 no. 9, pp. 1662-1673, 2014

[23] J. Jin, Y. R. Zheng, W. Chen, and C. Xiao, "Hybrid precoding for millimeter wave MIMO systems: A matrix factorization approach," IEEE Transactions on Wireless Communications, vol. 17, no. 5, pp. 33273339, 2018.

[24] A. Tang and X. Wang, "A-duplex: Medium access control for efficient coexistence between full-duplex and half-duplex communications,' IEEE Transactions on Wireless Communications, vol. 14, no. 10, pp. 5871-5885, 2015

[25] J. P. González-Coma et al., "Channel estimation and hybrid precoding for frequency selective multiuser mmwave MIMO systems," IEEE Journal of Selected Topics in Signal Processing, vol. 12, no. 2, pp. 353-367, 2018.

[26] K. Satyanarayana et al., "Hybrid beamforming design for full-duplex millimeter wave communication," IEEE Transactions on Vehicular Technology, vol. 68 , no. 2, pp. 1394-1404, 2018.
[27] J. Jang and K. B. Lee, "Transmit power adaptation for multiuser OFDM systems," IEEE Journal on selected areas in communications, vol. 21, no. 2, pp. 171-178, 2003.

[28] A. Sabharwal, P. Schniter, D. Guo, D. W. Bliss, S. Rangarajan, and R. Wichman, "In-band full-duplex wireless: Challenges and opportunities," IEEE Journal on selected areas in communications, vol. 32, no. 9, pp. 1637-1652, 2014.

[29] E. G. Larsson et al., "Massive MIMO for next generation wireless systems," vol. 52, no. 2, pp. 186-195, 2014.

[30] K. E. Baddour and N. C. Beaulieu, "Autoregressive modeling for fading channel simulation," IEEE Transactions on Wireless Communications, vol. 4, no. 4, pp. 1650-1662, 2005.

[31] J. Gao and H. Liu, "Decision-directed estimation of MIMO time-varying rayleigh fading channels," IEEE Transactions on wireless communications, vol. 4, no. 4, pp. 1412-1417, 2005.

[32] E. Everett, C. Shepard, L. Zhong, and A. Sabharwal, "Softnull: Manyantenna full-duplex wireless via digital beamforming," IEEE Transac tions on Wireless Communications, vol. 15, no. 12, pp. 8077-8092, 2016.

[33] Z. Shen, J. G. Andrews, and B. L. Evans, "Adaptive resource allocation in multiuser OFDM systems with proportional rate constraints," IEEE transactions on wireless communications, vol. 4, no. 6, pp. 2726-2737, 2005.

[34] S. Boyd, S. P. Boyd, and L. Vandenberghe, Convex optimization. Cambridge university press, 2004.

[35] G. Scutari, D. P. Palomar, and S. Barbarossa, "The MIMO iterative waterfilling algorithm," IEEE Transactions on Signal Processing, vol. 57, no. 5, pp. 1917-1935, 2009.

[36] C. Rusu, R. Mendez-Rial, N. González-Prelcic, and R. W. Heath, "Low complexity hybrid precoding strategies for millimeter wave communication systems," IEEE Transactions on Wireless Communications, vol. 15 , no. 12, pp. 8380-8393, 2016.

[37] J. Lee and S. Leyffer, Mixed integer nonlinear programming. Springer Science \& Business Media, 2011, vol. 154.

[38] F. Sohrabi et al., "Hybrid digital and analog beamforming design for large-scale antenna arrays," IEEE Journal of Selected Topics in Signal Processing, vol. 10, no. 3, pp. 501-513, 2016.

[39] F. Sohrabi and W. Yu, "Hybrid analog and digital beamforming for mmwave OFDM large-scale antenna arrays," IEEE Journal on Selected Areas in Communications, vol. 35, no. 7, pp. 1432-1443, 2017.

[40] D. P. Palomar and J. R. Fonollosa, "Practical algorithms for a family of waterfilling solutions," IEEE transactions on Signal Processing, vol. 53, no. 2, pp. 686-695, 2005. 